\title{
Some applications of Gröbner bases in homological algebra
}

\author{
Oswaldo Lezama \\ Grupo de Álgebra Conmutativa Computacional - SAC ${ }^{2}$ \\ Departamento de Matemáticas, Universidad Nacional de Colombia, Bogotá, Colombia \\ E-mail address: jolezamas@unal.edu.co
}

\begin{abstract}
In this paper we make some computations in homological algebra using Gröbner bases for modules over polynomials rings with coefficients in a Noetherian commutative ring. In particular, we show easy procedures for computing the Ext and Tor modules.

Key words and phrases: Gröbner bases, syzygies, finite presentation of a module, free resolutions, Ext, Tor.

2000 Mathematics Subject Classification: Primary: 13P10. Secondary: 13D02, $13 \mathrm{D} 07$.
\end{abstract}

\section{Introduction}

Many concepts in algebra are easy to formulate but in concrete situations it is not easy to make computations involving them. For example, if $M$ is a submodule of the free module $A^{m}$, where $A=R\left[x_{1}, \ldots, x_{n}\right]$ and $R$ is a Noetherian commutative ring, a presentation of $M$ is defined by the quotient $A^{s} / K$, where $s$ is the number of generators of $M$ and $K$ is the kernel of the canonical homomorphism $A^{s} \longrightarrow M$ that sends the canonical vectors of $A^{s}$ into the generators of $M$. However, for concrete examples the computation of $K$ is not an easy task. For instance, if $M=<\boldsymbol{f}_{1}, \boldsymbol{f}_{2}>\subseteq\left(\mathbb{Z}_{10}[x, y]\right)^{2}$, where $\boldsymbol{f}_{1}=\left(3 x^{2} y+3 x, x y-2 y\right)$ and $\boldsymbol{f}_{2}=\left(7 x y^{2}+y, y^{2}-4 x\right)$ then a direct computation of $K$ without a Computer Algebra System, or at least without an algorithmic procedure, is a very extensive an complicated exercise. Perhaps the most important algorithmic tools for solving this kind of problems are the Gröbner bases introduced in the sixties by Bruno Buchberger (see [3]). Gröbner bases play a key role in computational commutative algebra and let us to solve many problems that are practically impossible to attack with traditional theoretical methods. 
Gröbner bases have been studied intensively in the last years, and there are a lot of interesting applications in many branches of mathematics such as in homological algebra, commutative algebra, algebraic geometry, differential algebra, graph theory, etc. Gröbner bases have been also used in applied sciences as statistics ([4]), robotic ([6]), linear control systems ([5],[14]), etc. There are excellent monographs that study the theory and applications of Gröbner bases. In particular, we can mention the recent works of Lorenzo Robbiano and Martin Kreuzer (see [11],[12] and also [9]), the monograph of Pfister and Greuel ([8]) that includes the package SINGULAR ([10]), the monographs of Cox et al with applications in Algebraic Geometry ([6], [7]), the classical book of Becker and Weispfenning ([2]), and the textbook of Adams and Loustaunau ([1]).

In this paper we present some applications of Gröbner bases of modules in homological algebra. Thus, if $R$ is a Noetherian commutative ring, $A=R\left[x_{1}, \ldots, x_{n}\right]$, and $A^{m}$ is the free module of vector columns of length $m \geq 1$ with entries in $R\left[x_{1}, \ldots, x_{n}\right]$, we will compute presentations of $\operatorname{Hom}_{A}(M, N), M \otimes_{A} N, \operatorname{Ext}_{A}^{r}(M, N)$ and $\operatorname{Tor}_{r}^{A}(M, N)$, where $M$ is a given submodule of $A^{m}$ and $N$ is a given submodule of $A^{l}$, with $m, l \geq 1$ and $r \geq 0$. The technique we will use is very simple, we compute presentations of submodules of $A^{m}$ using syzygies and Gröbner bases, and with this, we compute free resolutions and the correspondent modules of homology.

The theory and methods of Gröbner bases for modules are well known. For example, in [1] is presented the theory of Gröbner bases for ideals of $A$ and for submodules of $\left(K\left[x_{1}, \ldots, x_{n}\right]\right)^{m}$, where $K$ is a field (see also the Chapter 2 of [8], and Chapters 2 and 3 of [11]). In [13] and [15] the theory was extended for submodules of $A^{m}$, in particular, in [13] was presented and proved the algorithm of Buchbeger for computing Gröbner bases of submodules of $A^{m}$. In the present paper we will use the Buchberger's algorithm of [13] for computing all Gröbner bases needed in the examples that illustrate our results and procedures below. Moreover, we will use the usual terminology about monomial orders on $A$ and $A^{m}$ (see [1] and [8]), in particular, we will use the POTREV order on monomials of $A^{m}$ defined as in [13], i.e., given a monomial order $>$ on $\operatorname{Mon}(A)$ (monomials of $A$ ), we define the following natural order on $\operatorname{Mon}\left(A^{m}\right)$ (monomials of $A^{m}$ ).

Definition 1. Let $\boldsymbol{X}=X \boldsymbol{e}_{i}$ and $\boldsymbol{Y}=Y \boldsymbol{e}_{j} \in \operatorname{Mon}\left(A^{m}\right)$, where $X, Y \in$ $\operatorname{Mon}(A)$ and $\left\{\boldsymbol{e}_{1}, \ldots, \boldsymbol{e}_{m}\right\}$ is the canonical basis of $A^{m}$. The POTREV order is defined by

$$
\boldsymbol{X}>\boldsymbol{Y} \Longleftrightarrow\left\{\begin{array}{l}
i<j \\
\text { or } \\
i=j \text { and } \quad X>Y .
\end{array}\right.
$$

São Paulo J.Math.Sci. 3, 1 (2009), 25-59 
Note that for this monomial order

$$
e_{1}>e_{2}>\cdots>e_{m}
$$

If $\boldsymbol{f} \neq \mathbf{0}$ is a vector of $A^{m}$, then we may write $\boldsymbol{f}$ as a sum of terms in the following way

$$
\boldsymbol{f}=c_{1} \boldsymbol{X}_{1}+\cdots+c_{t} \boldsymbol{X}_{t},
$$

where $c_{1}, \ldots, c_{t} \in R-0$ and $\boldsymbol{X}_{1}>\boldsymbol{X}_{2}>\cdots>\boldsymbol{X}_{t}$ are monomials of $\operatorname{Mon}\left(A^{m}\right)$.

Definition 2. With the above notation, we say that

(i) $l t(f)=c_{1} \boldsymbol{X}_{1}$ is the leading term of $\boldsymbol{f}$.

(ii) $l c(\boldsymbol{f})=c_{1}$ is the leading coefficient of $\boldsymbol{f}$.

(iii) $\operatorname{lm}(\boldsymbol{f})=\boldsymbol{X}_{1}$ is the leading monomial of $\boldsymbol{f}$.

With the above terminology we recall the definition of Gröbner basis for submodules of $A^{m}$ (see [13]): Let $G \neq 0$ be a non empty subset of $A^{m}$, then

$$
\operatorname{Lt}(G)=<l t(\boldsymbol{g}) \mid \boldsymbol{g} \in G>
$$

is the submodule of $A^{m}$ generated for the leading terms of vectors of $G$. Let $M \neq 0$ be a submodule of $A^{m}$ and let $G \neq 0$ be a non empty finite subset of $M$, we say that $G$ is a Gröbner basis for $M$ if $\operatorname{Lt}(M)=\operatorname{Lt}(G)$. Taking $m=1$ we get the definition of Gröbner basis for ideals of $A$. An effective method for computing Gröbner bases of ideals and submodules over commutative polynomials rings is the the Buchberger's algorithm that can be found in [1] or [13]. For more details about the general theory of Gröbner bases of ideals and modules see [1], [8] and [13].

The paper in divided in four sections. The second section is dedicated to compute syzygies of modules, the presentation of a given module and the kernel and the image of a homomorphism between modules. In the third section we compute the modules $\operatorname{Hom}_{A}(M, N)$ and $M \otimes_{A} N$, and also we will compute a free resolution of a given submodule $M$ of $A^{m}$. The last section is dedicated to compute presentations for $\operatorname{Ext}_{A}^{r}(M, N)$ and $\operatorname{Tor}_{r}^{A}(M, N)$. All computations will be illustrated with examples. We remark that in the literature is difficult to find such illustrative examples.

\section{Elementary applications of Gröbnes bases}

In this section we will list the most basic applications of Gröbner bases in module theory that we will use later. We will compute syzygies of modules, the presentation of a given module, the kernel and the image of a homomorphism between modules. All of these computations are analogues of those for ideals of $R\left[x_{1}, \ldots, x_{n}\right]$ or submodules of $K\left[x_{1}, \ldots, x_{n}\right]^{m}$, and 
the proofs have been adapted (see Chapters 3 and 4 in [1], and Chapter 2 of $[8])$.

2.1. Syzygy of a module. We start computing the syzygy of a submodule $M=<\boldsymbol{f}_{1}, \ldots, \boldsymbol{f}_{s}>$ of $A^{m}$. For this we consider the matrix $F=\left[\boldsymbol{f}_{1} \cdots \boldsymbol{f}_{s}\right]$ and we recall that $\operatorname{Syz}(F)$ consists of vectors $\boldsymbol{h}=\left(h_{1}, \ldots, h_{s}\right) \in A^{s}$ such that

$$
h_{1} \boldsymbol{f}_{1}+\cdots+h_{s} \boldsymbol{f}_{s}=\mathbf{0},
$$

i.e., $F \boldsymbol{h}=\mathbf{0}$. We note that $S y z(F)$ is a submodule of $A^{s}$ and we also define $\operatorname{Syz}(M):=\operatorname{Syz}(F)$.

In order to compute $S y z(M)$ we need the following preliminary result (see Lemma 2.8.2 in [8]).

Theorem 3. Let $F=\left\{\boldsymbol{f}_{1}, \ldots, \boldsymbol{f}_{s}\right\}$ be a set of non-zero vectors of $A^{m}$ and $G$ a Gröbner basis for $M=<F>$ with respect to POTREV order on $\operatorname{Mon}\left(A^{m}\right)$. Then, for any $k=0, \ldots, m-1, G \cap\left(\bigoplus_{i=k+1}^{m} A \boldsymbol{e}_{i}\right)$ is a Gröbner basis for $M \cap\left(\bigoplus_{i=k+1}^{m} A \boldsymbol{e}_{i}\right)$.

The key for computing syzygies is the following theorem.

Theorem 4. Let $F=\left\{\boldsymbol{f}_{1}, \ldots, \boldsymbol{f}_{s}\right\}$ be a set of non-zero vectors of $A^{m}$. Consider the canonical embedding

$$
A^{m} \longrightarrow A^{m+s}
$$

and the canonical projection

$$
\pi: A^{m+s} \longrightarrow A^{s} .
$$

Let $G=\left\{\boldsymbol{g}_{1}, \ldots, \boldsymbol{g}_{t}\right\}$ be a Gröbner basis for $<\boldsymbol{f}_{1}+\boldsymbol{e}_{m+1}, \ldots, \boldsymbol{f}_{s}+\boldsymbol{e}_{m+s}>$ with respect to the POTREV order on $M$ on $\left(A^{m+s}\right)$. If $G \cap\left(\bigoplus_{i=m+1}^{m+s} A \boldsymbol{e}_{i}\right)=$ $\left\{\boldsymbol{g}_{1}, \ldots, \boldsymbol{g}_{l}\right\}$, then $\operatorname{Syz}(F)=<\pi\left(\boldsymbol{g}_{1}\right), \ldots, \pi\left(\boldsymbol{g}_{l}\right)>$.

Proof. If $K$ is a field the proof of the Lemma 2.5.3 of [8] for $A=$ $K\left[x_{1}, \ldots, x_{n}\right]$ applies in our general situation. We will adapt this proof using a matrix notation. Let $M=<\boldsymbol{f}_{1}+\boldsymbol{e}_{m+1}, \ldots, \boldsymbol{f}_{s}+\boldsymbol{e}_{m+s}>$, by the previous theorem $\left\{\boldsymbol{g}_{1}, \ldots, \boldsymbol{g}_{l}\right\}$ is a Gröbner basis for $M \cap\left(\bigoplus_{i=m+1}^{m+s} A \mathbf{e}_{i}\right)$. For $1 \leq v \leq l$, let

$$
\boldsymbol{g}_{v}=\left[\begin{array}{c}
0 \\
\vdots \\
0 \\
g_{m+1, v} \\
\vdots \\
g_{m+s, v}
\end{array}\right]
$$


We know that during the process of computing a Gröbner basis we can express each $\boldsymbol{g}_{i}$ as a combination of generators of $M$ (see [13]), thus there exist elements $h_{1}, \ldots, h_{s} \in A$ such that

$$
\boldsymbol{g}_{v}=h_{1}\left[\begin{array}{c}
f_{11} \\
\vdots \\
f_{m 1} \\
1 \\
0 \\
\vdots \\
0
\end{array}\right]+\cdots+h_{s}\left[\begin{array}{c}
f_{1 s} \\
\vdots \\
f_{m s} \\
0 \\
0 \\
\vdots \\
1
\end{array}\right] \text {, }
$$

where by the canonical embedding

$$
\boldsymbol{f}_{i}=\left[\begin{array}{c}
f_{1 i} \\
\vdots \\
f_{m i} \\
0 \\
\vdots \\
1 \\
\vdots \\
0
\end{array}\right]
$$

then $h_{i}=g_{m+i, v}$ for each $1 \leq i \leq s$ and also

$$
h_{1}\left[\begin{array}{c}
f_{11} \\
\vdots \\
f_{m 1}
\end{array}\right]+\cdots+h_{s}\left[\begin{array}{c}
f_{1 s} \\
\vdots \\
f_{m s}
\end{array}\right]=0
$$

i.e., $g_{m+1, v} \boldsymbol{f}_{1}+\cdots+g_{m+s, v} \boldsymbol{f}_{s}=0$, and hence, $\pi\left(\boldsymbol{g}_{v}\right) \in \operatorname{Syz}(F)$ for each $1 \leq v \leq l$. This proves that $<\pi\left(\boldsymbol{g}_{1}\right), \ldots, \pi\left(\boldsymbol{g}_{l}\right)>\subseteq \operatorname{Syz}(F)$.

Conversely, let $\boldsymbol{h}=\left(h_{1}, \ldots, h_{s}\right) \in \operatorname{Syz}(F)$, then $h_{1} \boldsymbol{f}_{1}+\cdots+h_{s} \boldsymbol{f}_{s}=\mathbf{0}$ and hence 


$$
h_{1}\left[\begin{array}{c}
f_{11} \\
\vdots \\
f_{m 1} \\
1 \\
0 \\
\vdots \\
0
\end{array}\right]+\cdots+h_{s}\left[\begin{array}{c}
f_{1 s} \\
\vdots \\
f_{m s} \\
0 \\
0 \\
\vdots \\
1
\end{array}\right]=\left[\begin{array}{c}
0 \\
\vdots \\
0 \\
h_{1} \\
h_{2} \\
\vdots \\
h_{s}
\end{array}\right] \in M \cap\left(\bigoplus_{i=m+1}^{m+s} A \mathbf{e}_{i}\right)
$$

Since $\left\{\boldsymbol{g}_{1}, \ldots, \boldsymbol{g}_{l}\right\}$ is a Gröbner basis for $M \cap\left(\bigoplus_{i=m+1}^{m+s} A \mathbf{e}_{i}\right)$, there exist $p_{1}, \ldots, p_{l} \in A$ such that

$$
\left[\begin{array}{c}
0 \\
\vdots \\
0 \\
h_{1} \\
\vdots \\
h_{s}
\end{array}\right]=p_{1}\left[\begin{array}{c}
0 \\
\vdots \\
0 \\
g_{m+1,1} \\
\vdots \\
g_{m+s, 1}
\end{array}\right]+\cdots+p_{l}\left[\begin{array}{c}
0 \\
\vdots \\
0 \\
g_{m+1, l} \\
\vdots \\
g_{m+s, l}
\end{array}\right],
$$

hence $\boldsymbol{h} \in<\pi\left(\boldsymbol{g}_{1}\right), \ldots, \pi\left(\boldsymbol{g}_{l}\right)>$. This complete the proof.

The following example illustrates the elimination method described in the previous theorem.

Example 5. In [13] we computed a Gröbner basis for $M=<\boldsymbol{f}_{1}, \boldsymbol{f}_{2}>$ with respect to POTREV order on $\operatorname{Mon}\left(A^{2}\right)$, where $\boldsymbol{f}_{1}=3 i x^{2} y \boldsymbol{e}_{1}+(1+$ i) $z \boldsymbol{e}_{1}+2 i x y^{2} \boldsymbol{e}_{2}+5 z \boldsymbol{e}_{2}, \boldsymbol{f}_{2}=(2+i) x^{2} z \boldsymbol{e}_{1}+y \boldsymbol{e}_{1}+3 x y^{2} \boldsymbol{e}_{2}+y^{2} \boldsymbol{e}_{2}+4 i z \boldsymbol{e}_{2}$ and $A=\mathbb{Z}[i][x, y, z]$. On Mon $(A)$ we used the order deglex with $x>y>z$. The Gröbner basis we computed is $G=\left\{\boldsymbol{f}_{1}, \boldsymbol{f}_{2}, \boldsymbol{f}_{3}, \boldsymbol{f}_{4}\right\}$, where $\boldsymbol{f}_{3}=3 y^{2} \boldsymbol{e}_{1}+$ $(-3+i) z^{2} \boldsymbol{e}_{1}+9 x y^{3} \boldsymbol{e}_{2}+(-4-2 i) x y^{2} z \boldsymbol{e}_{2}+3 y^{3} \boldsymbol{e}_{2}+12 i y z \boldsymbol{e}_{2}+(-5+10 i) z^{2} \boldsymbol{e}_{2}$ and $\boldsymbol{f}_{4}=9 x^{3} y^{3} \boldsymbol{e}_{2}+(-4-2 i) x^{3} y^{2} z \boldsymbol{e}_{2}+3 x^{2} y^{3} \boldsymbol{e}_{2}+12 i x^{2} y z \boldsymbol{e}_{2}+(-5+$ 10i) $x^{2} z^{2} \boldsymbol{e}_{2}-2 x y^{3} \boldsymbol{e}_{2}+(3-3 i) x y^{2} z \boldsymbol{e}_{2}+(1-i) y^{2} z \boldsymbol{e}_{2}+5 i y z \boldsymbol{e}_{2}+(4+4 i) z^{2} \boldsymbol{e}_{2}$. Using again the algorithm of Buchberger presented in the Theorem 23 of [13] we compute a Gröbner basis $G^{\prime}$ for $F^{\prime}=\left\{\boldsymbol{f}_{1}+\boldsymbol{e}_{3}, \boldsymbol{f}_{2}+\boldsymbol{e}_{4}\right\}$,

$$
G^{\prime}=\left\{\boldsymbol{g}_{1}^{\prime}, \boldsymbol{g}_{2}^{\prime}, \boldsymbol{g}_{3}^{\prime}, \boldsymbol{g}_{4}^{\prime}\right\}
$$

where $\boldsymbol{g}_{1}^{\prime}=\boldsymbol{f}_{1}+\boldsymbol{e}_{3}, \boldsymbol{g}_{2}^{\prime}=\boldsymbol{f}_{2}+\boldsymbol{e}_{4}, \boldsymbol{g}_{3}^{\prime}=\boldsymbol{f}_{3}+(-1+2 i) z \boldsymbol{e}_{3}+3 y \boldsymbol{e}_{4}$ and $\boldsymbol{g}_{4}^{\prime}=\boldsymbol{f}_{4}+(-1+2 i) x^{2} z \boldsymbol{e}_{3}+i y \boldsymbol{e}_{3}+3 x^{2} y \boldsymbol{e}_{4}+(1-i) z \boldsymbol{e}_{4}$. We observe that $G^{\prime} \cap\left(A e_{3} \oplus A e_{4}\right)=\emptyset$, and hence $\operatorname{Syz}\left(\boldsymbol{f}_{1}, \boldsymbol{f}_{2}\right)=0$. 
2.2. Presentation of a module. Let $M=<\boldsymbol{f}_{1}, \ldots, \boldsymbol{f}_{s}>$ be a submodule of $A^{m}$, there exists a natural surjective homomorphism $\pi_{M}: A^{s} \longrightarrow M$ defined by $\pi_{M}\left(\boldsymbol{e}_{i}\right)=\boldsymbol{f}_{i}$, where $\left\{\boldsymbol{e}_{i}\right\}_{1 \leq i \leq s}$ is the canonical basis of $A^{s}$. If $K_{M}=\operatorname{ker}\left(\pi_{M}\right)$, then we have the isomorphism $\overline{\pi_{M}}: A^{s} / K_{M} \cong M$, defined by $\overline{\pi_{M}}\left(\overline{\boldsymbol{e}_{i}}\right)=\boldsymbol{f}_{i}$, where $\overline{\boldsymbol{e}_{i}}=\boldsymbol{e}_{i}+K_{M}$. We note that $K_{M}=<h_{1}, \ldots, h_{s_{1}}>$ is also a finitely generated module and we have the exact sequence

$$
A^{s_{1}} \stackrel{\delta_{M}}{\longrightarrow} A^{s} \stackrel{\pi_{M}}{\longrightarrow} M \longrightarrow 0
$$

with $\delta_{M}=i_{M} \circ \pi_{M}^{\prime}$, where $i_{M}$ is the inclusion of $K_{M}$ in $A^{s}$ and $\pi_{M}^{\prime}$ is the natural surjective homomorphism from $A^{s_{1}}$ to $K_{M}$. We recall that the quotient module $A^{s} / K_{M}$, or equivalently, the exact sequence (2.1), is a presentation of $M$. We observe that $K_{M}=\operatorname{Syz}(M)=\operatorname{Syz}(F)$, where $F=$ $\left[\boldsymbol{f}_{1} \cdots \boldsymbol{f}_{s}\right]$, and consequently, the Theorem 4 gives a method for computing a presentation of a module. On the other hand, let $\Delta_{M}$ be the matrix of $\delta_{M}$ in the canonical bases of $A^{s_{1}}$ and $A^{s}$, then the columns of $\Delta_{M}$ are the generators of $\operatorname{Syz}(F)$ since $\operatorname{Im}\left(\delta_{M}\right)=\operatorname{ker}\left(\pi_{M}\right)$. We will also say that $\Delta_{M}$ is a matrix presentation of $M$.

The next example will be used often in this paper.

Example 6. Let $M=<\boldsymbol{f}_{1}, \boldsymbol{f}_{2}>\subseteq\left(\mathbb{Z}_{10}[x, y]\right)^{2}$ and $N=<\boldsymbol{g}_{1}, \boldsymbol{g}_{2}, \boldsymbol{g}_{3}>\subseteq$ $\left(\mathbb{Z}_{10}[x, y]\right)^{2}$, where $\boldsymbol{f}_{1}=\left(3 x^{2} y+3 x, x y-2 y\right), \boldsymbol{f}_{2}=\left(7 x y^{2}+y, y^{2}-4 x\right), \boldsymbol{g}_{1}=$ $(0, x)$ and $\boldsymbol{g}_{2}=(y, x)$ and $\boldsymbol{g}_{3}=(2 x, x)$, then applying the Theorem 4 with order deglex on $\operatorname{Mon}(\mathbb{Z}[x, y])$ and $x>y$, we get presentations for $M$ and $N$,

$$
M \cong A^{2} / \operatorname{Syz}(M), N \cong A^{3} / \operatorname{Syz}(N)
$$

where

$$
\operatorname{Syz}(M)=<(5 y, 5 x)>, \operatorname{Syz}(N)=<(5,0,5),(2 x+9 y, 8 x, y)>.
$$

Presentations of quotient modules could be also computed. In fact, let $N \subseteq M$ be submodules of $A^{m}, M=<\boldsymbol{f}_{1}, \ldots, \boldsymbol{f}_{s}>, N=<\boldsymbol{g}_{1}, \ldots, \boldsymbol{g}_{t}>$, $M / N=<\overline{\boldsymbol{f}_{1}}, \ldots, \overline{\boldsymbol{f}_{s}}>$, then we have a canonical surjective homomorphism $\delta: A^{s} \longrightarrow M / N$ such that a presentation of $M / N$ is given by $M / N \cong$ $A^{s} / \operatorname{Syz}(M / N)$. But $\operatorname{Syz}(M / N)$ can be computed in the following way. $\boldsymbol{h}=$ $\left(h_{1}, \ldots, h_{s}\right) \in \operatorname{Syz}(M / N)$ if and only if $h_{1} \boldsymbol{f}_{1}+\cdots+h_{s} \boldsymbol{f}_{s} \in<\boldsymbol{g}_{1}, \ldots, \boldsymbol{g}_{t}>$ if and only if there exist $h_{s+1}, \ldots, h_{s+t} \in A$ such that $h_{1} \boldsymbol{f}_{1}+\cdots+h_{s} \boldsymbol{f}_{s}+$ $h_{s+1} \boldsymbol{g}_{1}+\cdots+h_{s+t} \boldsymbol{g}_{t}=\mathbf{0}$ if and only if $\left(h_{1}, \ldots, h_{s}, h_{s+1}, \ldots, h_{s+t}\right) \in$ $\operatorname{Syz}(H)$, where

$$
H=\left[\begin{array}{llllll}
\boldsymbol{f}_{1} & \cdots & \boldsymbol{f}_{s} & \boldsymbol{g}_{1} & \cdots & \boldsymbol{g}_{t}
\end{array}\right] .
$$

Thus we have the following well know result. 
Theorem 7. With the notation above, a presentation of $M / N$ is given by $A^{s} / \operatorname{Syz}(M / N)$, where a set of generators of $S y z(M / N)$ are the first $s$ coordinates of generators of $\operatorname{Syz}(H)$.

2.3. Kernel and image of a homomorphism. Let $M \subseteq A^{m}$ and $N \subseteq A^{l}$ be modules, $M=<\boldsymbol{f}_{1}, \ldots, \boldsymbol{f}_{s}>, N=<\boldsymbol{g}_{1}, \ldots, \boldsymbol{g}_{t}>$, and let $\phi: M \longrightarrow N$ be a homomorphism. Then, there exists a matrix $\Phi=\left[\phi_{j i}\right]$ of size $t \times s$ with entries in $A$ defined by

$$
\phi\left(\boldsymbol{f}_{i}\right)=\phi_{1 i} \boldsymbol{g}_{1}+\cdots+\phi_{t i} \boldsymbol{g}_{t},
$$

for each $1 \leq i \leq s$. We compute now a system of generators and a presentation for $\operatorname{ker} \phi$ and $\operatorname{Im}(\phi)$. We assume that the homomorphism $\phi$ is well-defined and it is given by the matrix $\Phi$. Using the notation of the previous subsection, let $A^{s} / \operatorname{Syz}(M)$ and $A^{t} / \operatorname{Syz}(N)$ be presentations of $M$ and $N$. We consider the canonical isomorphisms

$$
\overline{\pi_{M}}: A^{s} / \operatorname{Syz}(M) \longrightarrow M, \overline{\pi_{N}}: A^{t} / \operatorname{Syz}(N) \longrightarrow N
$$

defined by $\overline{\pi_{M}}\left(\overline{\boldsymbol{e}_{i}}\right)=\boldsymbol{f}_{i}, 1 \leq i \leq s, \overline{\pi_{N}}\left(\overline{\boldsymbol{e}_{j}^{\prime}}\right)=\boldsymbol{g}_{j}, 1 \leq j \leq t$, where $\left\{\boldsymbol{e}_{i}\right\}_{1 \leq i \leq s}$ is the canonical basis of $A^{s}$ and $\left\{\boldsymbol{e}_{j}^{\prime}\right\}_{1 \leq j \leq t}$ is the canonical basis of $A^{t}$. Then, we have the following commutative diagram

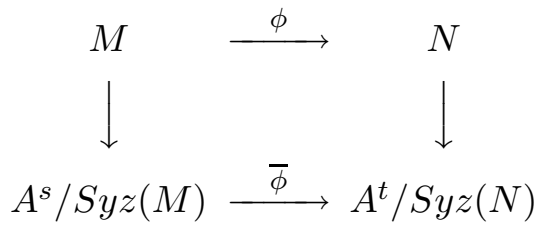

where the vertical arrows are the isomorphisms $\left(\overline{\pi_{M}}\right)^{-1}$ and $\left(\overline{\pi_{N}}\right)^{-1}$. Hence, $\bar{\phi}\left(\overline{\boldsymbol{e}_{i}}\right)=\left(\overline{\pi_{N}}\right)^{-1} \circ \phi \circ \overline{\pi_{M}}\left(\overline{\boldsymbol{e}_{i}}\right)=\phi_{1 i} \overline{\boldsymbol{e}_{1}^{\prime}} \cdots+\phi_{t i} \overline{\boldsymbol{e}_{t}^{\prime}}$, for each $1 \leq i \leq s$. We observe that the matrix of $\bar{\phi}$ coincides with the matrix of $\phi, \operatorname{ker}(\phi) \cong \operatorname{ker}(\bar{\phi})$ and $\operatorname{Im}(\phi) \cong \operatorname{Im}(\bar{\phi})$.

Let $h_{1} \boldsymbol{f}_{1}+\cdots+h_{s} \boldsymbol{f}_{s} \in \operatorname{ker}(\phi)$, then $\left(\overline{\pi_{N}}\right)^{-1}\left(\phi\left(h_{1} \boldsymbol{f}_{1}+\cdots+h_{s} \boldsymbol{f}_{s}\right)\right)=$ $\overline{\mathbf{0}}=\bar{\phi}\left(\left(\overline{\pi_{M}}\right)^{-1}\left(h_{1} \boldsymbol{f}_{1}+\cdots+h_{s} \boldsymbol{f}_{s}\right)\right)=\bar{\phi}\left(h_{1} \overline{\boldsymbol{e}_{1}}+\cdots+h_{s} \overline{\boldsymbol{e}_{s}}\right)=h_{1} \bar{\phi}\left(\overline{\boldsymbol{e}_{1}}\right)+$ $\cdots+h_{s} \bar{\phi}\left(\overline{\boldsymbol{e}_{s}}\right)=h_{1}\left(\phi_{11} \overline{\boldsymbol{e}_{1}^{\prime}}+\cdots+\phi_{t 1} \overline{\boldsymbol{e}_{t}^{\prime}}\right)+\cdots+h_{s}\left(\phi_{1 s} \overline{\boldsymbol{e}_{1}^{\prime}}+\cdots+\phi_{t s} \overline{\boldsymbol{e}_{t}^{\prime}}\right)=$ $\left(h_{1} \phi_{11}+\cdots+h_{s} \phi_{1 s}\right) \overline{\boldsymbol{e}_{1}^{\prime}}+\cdots+\left(h_{1} \phi_{t 1}+\cdots+h_{s} \phi_{t s}\right) \overline{\boldsymbol{e}_{t}^{\prime}}$. This implies that $\left(h_{1} \phi_{11}+\cdots+h_{s} \phi_{1 s}\right) \boldsymbol{e}_{1}^{\prime}+\cdots+\left(h_{1} \phi_{t 1}+\cdots+h_{s} \phi_{t s}\right) \boldsymbol{e}_{t}^{\prime} \in \operatorname{Syz}(N)$. We assume that we have computed a system of generators for $\operatorname{Syz}(N)=<$ $\boldsymbol{u}_{1}, \ldots, \boldsymbol{u}_{t_{1}}>\subseteq A^{t}$ (Theorem 4 ). Hence, there exist $h_{s+1}, \ldots, h_{s+t_{1}} \in A$ such that 


$$
h_{1}\left[\begin{array}{c}
\phi_{11} \\
\vdots \\
\phi_{t 1}
\end{array}\right]+\cdots+h_{s}\left[\begin{array}{c}
\phi_{1 s} \\
\vdots \\
\phi_{t s}
\end{array}\right]+h_{s+1} \boldsymbol{u}_{1}+\cdots+h_{s+t_{1}} \boldsymbol{u}_{t_{1}}=\mathbf{0}
$$

From these computations we also can conclude that

$$
h_{1} \boldsymbol{f}_{1}+\cdots+h_{s} \boldsymbol{f}_{s} \in \operatorname{ker}(\phi) \Leftrightarrow \overline{\left(h_{1}, \ldots, h_{s}\right)} \in \operatorname{ker}(\bar{\phi}) .
$$

Thus, we have proved the following theorem.

Theorem 8. With the notation above, let

$$
H=\left[\begin{array}{llllll}
\Phi_{1} & \cdots & \Phi_{s} & \boldsymbol{u}_{1} & \cdots & \boldsymbol{u}_{t_{1}}
\end{array}\right],
$$

where $\Phi_{i}$ is the $i^{\text {th }}$ column of the matrix $\Phi, 1 \leq i \leq s$. Then,

$$
\left(h_{1}, \ldots, h_{s}, h_{s+1}, \ldots, h_{s+t_{1}}\right) \in S y z(H) \Leftrightarrow h_{1} \boldsymbol{f}_{1}+\cdots+h_{s} \boldsymbol{f}_{s} \in \operatorname{ker}(\phi) .
$$

Thus, if $\left\{\boldsymbol{z}_{1}, \ldots, \boldsymbol{z}_{v}\right\} \subset A^{s+t_{1}}$ is a system of generators of Syz(H), let $\boldsymbol{z}_{k} \in$ $A^{s}$ be the vector obtained from $z_{k}$ when omitting the last $t_{1}$ components, $1 \leq k \leq v$, then $\left\{\overline{\boldsymbol{z}_{1}^{\prime}}, \ldots, \overline{\boldsymbol{z}_{v}^{\prime}}\right\}$ is a system of generators for $\operatorname{ker}(\bar{\phi})$. Moreover, if

$$
z_{1}^{\prime}=\left(h_{11}, \ldots, h_{1 s}\right), \ldots, z_{v}^{\prime}=\left(h_{v 1}, \ldots, h_{v s}\right),
$$

then $\left\{h_{11} f_{1}+\cdots+h_{1 s} f_{s}, \ldots, h_{v 1} f_{1}+\cdots+h_{v s} f_{s}\right\}$ is a system of generators for $\operatorname{ker}(\phi)$.

A presentation of $\operatorname{ker}(\phi)$ is given in the following way.

Corollary 9. With the notation of this section, a presentation of $\operatorname{ker}(\phi)$ is given by $A^{v} / K$, where

$$
K=\operatorname{Syz}(\operatorname{ker}(\phi))=\operatorname{Syz}\left[h_{11} \boldsymbol{f}_{1}+\cdots+h_{1 s} \boldsymbol{f}_{s} \quad \cdots \quad h_{v 1} \boldsymbol{f}_{1}+\cdots+h_{v s} \boldsymbol{f}_{s}\right] .
$$

Example 10. In the Step 6 of Section 3.1 we will prove that the function $\phi$ defined by

$$
\begin{aligned}
& M \stackrel{\phi}{\rightarrow} N \\
& \boldsymbol{f}_{1} \mapsto x \boldsymbol{g}_{3} \\
& \boldsymbol{f}_{2} \mapsto y \boldsymbol{g}_{1}
\end{aligned}
$$

is an homomorphism, where $M$ and $N$ are as in the Example 6 . Now we will calculate a system of generators for $\operatorname{ker}(\phi)$. We note that the matrix of $\phi$ is

$$
\Phi=\left[\begin{array}{ll}
0 & y \\
0 & 0 \\
x & 0
\end{array}\right]
$$


thus, we must compute $\operatorname{Syz}(H)$ where

$$
H=\left[\begin{array}{cccc}
0 & y & 5 & 2 x+9 y \\
0 & 0 & 0 & 8 x \\
x & 0 & 5 & y
\end{array}\right] .
$$

For this we apply the Theorem 4 and we get

$$
\operatorname{Syz}(H)=<(0,0,2,0),(0,0,-y, 5)>.
$$

By the Theorem 8 , we omit the last two components of $(0,0,2,0)$, $(0,0,-y, 5)$ and we get that $\operatorname{ker}(\phi)=0$.

An explicit presentation for $\operatorname{ker}(\bar{\phi})$ could be also given. We assume that we have computed a system of generators for $\operatorname{Syz}(M)=\left\langle\boldsymbol{w}_{1}, \ldots, \boldsymbol{w}_{s_{1}}\right\rangle \subseteq$ $A^{s}$. We know that a presentation of $\operatorname{ker}(\bar{\phi})$ is given by $\operatorname{ker}(\bar{\phi}) \cong A^{v} / K^{\prime}$, where $K^{\prime}=S y z(\operatorname{ker}(\bar{\phi}))=S y z\left(<\overline{\boldsymbol{z}_{1}^{\prime}}, \ldots, \overline{\boldsymbol{z}_{v}^{\prime}}>\right)$. But, $\left(l_{1}, \ldots, l_{v}\right) \in S y z(<$ $\left.\overline{\boldsymbol{z}_{1}^{\prime}}, \ldots, \overline{\boldsymbol{z}_{v}^{\prime}}>\right)$ if and only if there exist $l_{v+1}, \ldots, l_{v+s_{1}} \in A$ such that $l_{1} \boldsymbol{z}_{1}^{\prime}+$ $\cdots+l_{v} \boldsymbol{z}_{v}^{\prime}+l_{v+1} \boldsymbol{w}_{1}+\cdots+l_{v+s_{1}} \boldsymbol{w}_{s_{1}}=\mathbf{0}$. Thus, we have proved the following corollary.

Corollary 11. With the notation above, let

$$
L=\left[\begin{array}{llllll}
z_{1}^{\prime} & \cdots & z_{v}^{\prime} & \boldsymbol{w}_{1} & \cdots & \boldsymbol{w}_{s_{1}}
\end{array}\right]
$$

if $\left\{\boldsymbol{l}_{1}, \ldots, \boldsymbol{l}_{q}\right\} \subset A^{v+s_{1}}$ is a system of generators of $\operatorname{Syz}(L)$, let $\boldsymbol{l}_{k}^{\prime} \in A^{v}$ be the vector obtained from $\boldsymbol{l}_{k}$ when omitting the last $s_{1}$ components, $1 \leq k \leq q$, then $\left\{l_{1}^{\prime}, \ldots, l_{q}^{\prime}\right\}$ is a system of generators for $K^{\prime}$, and hence, a presentation of $\operatorname{ker}(\bar{\phi})$ is given by $A^{v} / K^{\prime}$.

We consider now the image of homomorphism $\phi: M \longrightarrow N$ in (2.2). Then the following result is clear from the above discussion.

Corollary 12. A system of generators for $\operatorname{Im}(\phi)$ is given by

$$
\operatorname{Im}(\phi)=<\phi_{11} \mathbf{g}_{1}+\cdots+\phi_{t 1} \mathbf{g}_{t}, \ldots, \phi_{1 s} \mathbf{g}_{1}+\cdots+\phi_{t s} \mathbf{g}_{t}>\text {. }
$$

A presentation of $\operatorname{Im}(\phi)$ is $A^{s} / I$, where

$$
I=S y z\left[\phi_{11} \mathbf{g}_{1}+\cdots+\phi_{t 1} \mathbf{g}_{t} \quad \ldots \quad \phi_{1 s} \mathbf{g}_{1}+\cdots+\phi_{t s} \mathbf{g}_{t}\right] .
$$

Example 13. Let $\phi$ be as in the Example 10, then by the previous corollary, $\operatorname{Im}(\phi)=<\left(2 x^{2}, x^{2}\right),(0, x y)>$. A presentation of $\operatorname{Im}(\phi)$ is given by $\operatorname{Syz}(I)$, where

$$
I=\left[\begin{array}{cc}
2 x^{2} & 0 \\
x^{2} & x y
\end{array}\right]
$$

so we apply again the Theorem 4 and we get $S y z(I)=<(5 y, 5 x)>$. Thus, $\operatorname{Im}(\phi) \cong A^{2} /<(5 y, 5 x)>$. This conclusion of course coincides with the results of the Examples 6 and 10 since $\operatorname{Im}(\phi) \cong M$. 
We conclude this section showing an explicit presentation of $\operatorname{Im}(\bar{\phi})$. We know that $\operatorname{Im}(\bar{\phi})=<\phi_{11} \overline{\boldsymbol{e}_{1}^{\prime}}+\cdots+\phi_{t 1} \overline{\boldsymbol{e}_{t}^{\prime}}, \ldots, \phi_{1 s} \overline{\boldsymbol{e}_{1}^{\prime}}+\cdots+\phi_{t s} \overline{\boldsymbol{e}_{t}^{\prime}}>$, thus a presentation of $\operatorname{Im}(\bar{\phi})$ is given by $\operatorname{Im}(\bar{\phi}) \cong A^{s} / \operatorname{Syz}(\operatorname{Im}(\bar{\phi}))$. Let $\left(h_{1}, \ldots, h_{s}\right) \in \operatorname{Syz}(\operatorname{Im}(\bar{\phi}))$, then there exist $h_{s+1}, \ldots, h_{s+t_{1}} \in A$ such that

$$
h_{1}\left[\begin{array}{c}
\phi_{11} \\
\vdots \\
\phi_{t 1}
\end{array}\right]+\cdots+h_{s}\left[\begin{array}{c}
\phi_{1 s} \\
\vdots \\
\phi_{t s}
\end{array}\right]+h_{s+1} \boldsymbol{u}_{1}+\cdots+h_{s+t_{1}} \boldsymbol{u}_{t_{1}}=\mathbf{0} .
$$

Thus, we have proved the following corollary.

Corollary 14. Let $H$ be the matrix in the Theorem 8. If $\left\{\boldsymbol{z}_{1}, \ldots, \boldsymbol{z}_{v}\right\} \subset$ $A^{s+t_{1}}$ is a system of generators of $\operatorname{Syz}(H)$, let $z_{k} \in A^{s}$ be the vector obtained from $z_{k}$ when omitting the last $t_{1}$ components, $1 \leq k \leq v$, then $\left\{z_{1}^{\prime}, \ldots, z_{v}^{\prime}\right\}$ is a system of generators for $\operatorname{Syz}(\operatorname{Im}(\bar{\phi}))$ and $\bar{A}^{s} / \operatorname{Syz}(\operatorname{Im}(\bar{\phi}))$ is a presentation of $\operatorname{Im}(\bar{\phi})$.

\section{Computing Hom, $\otimes$ and free resolutions}

The main goal of the paper is to compute the modules Ext and Tor, in this section we will compute the particular cases $\operatorname{Ext}_{A}^{0}(M, N)=$ $\operatorname{Hom}_{A}(M, N)$ and $\operatorname{Tor}_{0}^{A}(M, N)=M \otimes_{A} N$. We also show how to compute free resolutions.

3.1. Computation of Hom. In this subsection we show a procedure for computing $\operatorname{Hom}_{A}(M, N)$, where $M$ is a submodule of $A^{m}$ and $N$ is a submodule of $A^{l}$. By computing $\operatorname{Hom}_{A}(M, N)$ we mean to find a presentation of $\operatorname{Hom}_{A}(M, N)$ and to find an specific set of generators for $\operatorname{Hom}_{A}(M, N)$. For fields, i.e., when $A=K\left[x_{1}, \ldots, x_{n}\right]$, where $K$ is a field, the computation of $\operatorname{Hom}_{A}(M, N)$ can be found in [1], [8] and [11]. The constructions there apply to our more general situation when the ring of coefficients of $A$ is a Noetherian commutative ring $R$, however we will add the explicit definition of some homomorphisms and we will prove the commutativity of some diagrams omitted in the literature.

We will illustrate the theory and steps of the procedure through the following particular example: $M=<\boldsymbol{f}_{1}, \boldsymbol{f}_{2}>\subseteq\left(\mathbb{Z}_{10}[x, y]\right)^{2}$ and $N=<$ $\boldsymbol{g}_{1}, \boldsymbol{g}_{2}, \boldsymbol{g}_{3}>\subseteq\left(\mathbb{Z}_{10}[x, y]\right)^{2}$, where $\boldsymbol{f}_{1}=\left(3 x^{2} y+3 x, x y-2 y\right), \boldsymbol{f}_{2}=\left(7 x y^{2}+\right.$ $\left.y, y^{2}-4 x\right), \boldsymbol{g}_{1}=(0, x)$ and $\boldsymbol{g}_{2}=(y, x)$ and $\boldsymbol{g}_{3}=(2 x, x)$ (see the Example $6)$.

Let $M$ and $N$ be submodules of $A^{m}$ and $A^{l}$, respectively, then $M$ and $N$ are finitely generated modules, $M=<\boldsymbol{f}_{1}, \ldots, \boldsymbol{f}_{s}>, N=<\boldsymbol{g}_{1}, \ldots, \boldsymbol{g}_{t}>$. In our concrete example, $A=\mathbb{Z}_{10}[x, y]$, and we choose the POTREV order 
for the monomials of $A^{2}$. The order in $\operatorname{Mon}(A)$ is deglex with $x>y$. So, in this illustrative example, $R=\mathbb{Z}_{10}, A=\mathbb{Z}_{10}[x, y], m=2, s=2, l=2, t=3$. We divide the procedure in some steps.

Step 1. Presentations of $M$ and $N$. In order to compute a presentation of $\operatorname{Hom}_{A}(M, N)$ we first compute presentations of $M$ and $N$ as we saw in the second section:

$$
M \cong A^{s} / K_{M}, N \cong A^{t} / K_{N},
$$

where $K_{M}$ and $K_{N}$ are the kernels of natural homomorphisms $\pi_{M}: A^{s} \longrightarrow$ $M$ and $\pi_{N}: A^{t} \longrightarrow N$ defined by $\pi_{M}\left(\boldsymbol{e}_{i}\right)=\boldsymbol{f}_{i}, \pi_{N}\left(\boldsymbol{e}_{j}^{\prime}\right)=\boldsymbol{g}_{j}, 1 \leq i \leq s$, $1 \leq j \leq t\left(\left\{\boldsymbol{e}_{i}\right\}_{1 \leq i \leq s}\right.$ is the canonical basis of $A^{m}$ and $\left\{\boldsymbol{e}_{j}^{\prime}\right\}_{1 \leq j \leq t}$ is the canonical basis of $\left.A^{l}\right)$. Then, $\operatorname{Hom}_{A}(M, N) \cong \operatorname{Hom}_{A}\left(A^{s} / K_{M}, A^{t} / K_{N}\right)$, and we can compute a presentation and a system of generators of

$\operatorname{Hom}_{A}\left(A^{s} / K_{M}, A^{t} / K_{N}\right)$ instead of $\operatorname{Hom}_{A}(M, N)$. However, in the last step of the procedure we will use the system of generators of $\operatorname{Hom}_{A}\left(A^{s} / K_{M}, A^{t} / K_{N}\right)$ for giving an explicit system of generators of $\mathrm{Hom}_{A}(M, N)$.

We recall that $K_{M}$ and $K_{N}$ are computed by the syzygies of the matrices

$$
F_{M}=\left[\begin{array}{lll}
\boldsymbol{f}_{1} & \cdots & \boldsymbol{f}_{s}
\end{array}\right], F_{N}=\left[\begin{array}{lll}
\boldsymbol{g}_{1} & \cdots & \boldsymbol{g}_{t}
\end{array}\right],
$$

i.e., $K_{M}=S y z\left(F_{M}\right)=S y z(M), K_{N}=S y z\left(F_{N}\right)=S y z(N)$. In our example,

$$
M \cong A^{2} / \operatorname{Syz}(M), N \cong A^{3} / \operatorname{Syz}(N),
$$

and by the Example 6 ,

$$
\operatorname{Syz}(M)=<(5 y, 5 x)>, \operatorname{Syz}(N)=<(5,0,5),(2 x+9 y, 8 x, y)>.
$$

Hence,

$$
\begin{aligned}
\operatorname{Hom}_{A}( & M, N) \cong \\
& \cong \operatorname{Hom}_{A}\left(A^{2} /<(5 y, 5 x)>, A^{3} /<(5,0,5),(2 x+9 y, 8 x, y)>\right) .
\end{aligned}
$$

Step 2. $\operatorname{Hom}_{A}\left(A^{s} / \operatorname{Syz}(M), A^{t} / \operatorname{Syz}(N)\right)$ as a kernel. Since $R$ is a Noetherian ring, $\operatorname{Syz}(M)$ and $S y z(N)$ are finitely generated $A$-modules, $\operatorname{Syz}(M)$ is generated by $s_{1}$ elements and $\operatorname{Syz}(N)$ is generated by $t_{1}$ elements. Thus, we also have surjective homomorphisms $\pi_{M}^{\prime}: A^{s_{1}} \longrightarrow$ $\operatorname{Syz}(M)$ and $\pi_{N}^{\prime}: A^{t_{1}} \longrightarrow \operatorname{Syz}(N)$, and hence the following sequences are exact

$$
\begin{aligned}
& A^{s_{1}} \stackrel{\delta_{M}}{\longrightarrow} A^{s} \stackrel{j_{M}}{\longrightarrow} A^{s} / \operatorname{Syz}(M) \longrightarrow 0 \\
& A^{t_{1}} \stackrel{\delta_{N}}{\longrightarrow} A^{t} \stackrel{j_{N}}{\longrightarrow} A^{t} / \operatorname{Syz}(N) \longrightarrow 0
\end{aligned}
$$

where $\delta_{M}=i_{M} \circ \pi_{M}^{\prime}, \delta_{N}=i_{N} \circ \pi_{N}^{\prime}, i_{M}, i_{N}$ denote inclusions, and $j_{M}, j_{N}$ are natural homomorphisms. From (3.1) we get the exact sequence 


$$
\begin{gathered}
0 \rightarrow \operatorname{Hom}_{A}\left(A^{s} / \operatorname{Syz}(M), A^{t} / \operatorname{Syz}(N)\right) \stackrel{p}{\rightarrow} \operatorname{Hom}_{A}\left(A^{s}, A^{t} / \operatorname{Syz}(N)\right) \stackrel{d}{\rightarrow} \\
\operatorname{Hom}_{A}\left(A^{s_{1}}, A^{t} / \operatorname{Syz}(N)\right),
\end{gathered}
$$

where

$$
d(\alpha)=\alpha \circ \delta_{M} \quad \text { for } \quad \alpha \in \operatorname{Hom}_{A}\left(A^{s}, A^{t} / \operatorname{Syz}(N)\right)
$$

and $p$ is defined in the same way. Thus,

$$
\operatorname{Hom}_{A}\left(A^{s} / \operatorname{Syz}(M), A^{t} / \operatorname{Syz}(N)\right) \cong \operatorname{ker}(d) .
$$

Step 3. Computation of $\mathrm{Hom}_{A}\left(A^{s}, A^{t} / \operatorname{Syz}(N)\right)$ and $\operatorname{Hom}_{A}\left(A^{s_{1}}, A^{t} / \operatorname{Syz}(N)\right)$. According to (3.3), we must compute presentations of $\operatorname{Hom}_{A}\left(A^{s}, A^{t} / \operatorname{Syz}(N)\right)$ and $\operatorname{Hom}_{A}\left(A^{s_{1}}, A^{t} / \operatorname{Syz}(N)\right)$. Let $\Delta_{M}$ be the matriz presentation of $M$ and $\Delta_{N}$ the matrix presentation of $N$.

In our example, $s_{1}=1, t_{1}=2$ and

$$
\Delta_{M}=\left[\begin{array}{l}
5 y \\
5 x
\end{array}\right], \Delta_{N}=\left[\begin{array}{cc}
5 & 2 x+9 y \\
0 & 8 x \\
5 & y
\end{array}\right] .
$$

Let $M_{t_{1} s}(A)$ be the ring of matrices with $t_{1}$ rows and $s$ columns with entries in $A$, in the same way we define $M_{t s}(A)$. Then, from (3.2) we get the diagram

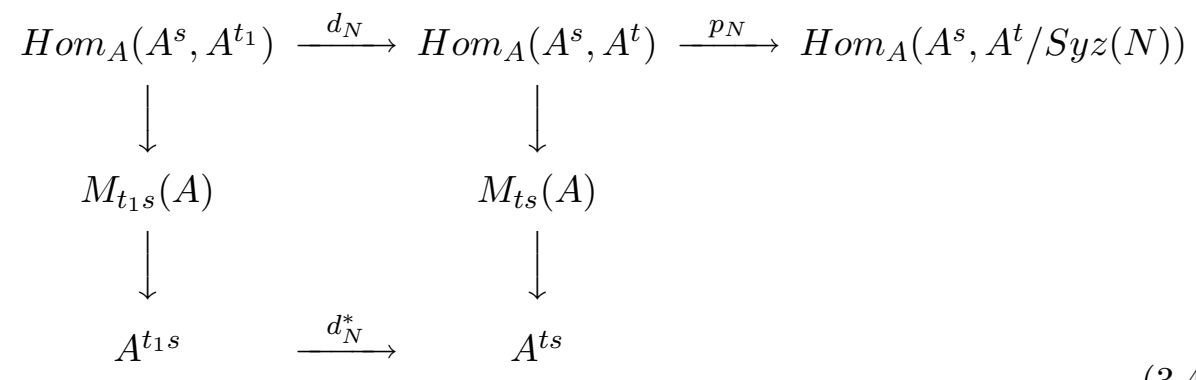

where $d_{N}$ is the natural homomorphism induced by $\delta_{N}$, i.e.,

$$
d_{N}(\beta)=\delta_{N} \circ \beta \text { for } \beta \in \operatorname{Hom}_{A}\left(A^{s}, A^{t_{1}}\right)
$$

and $p_{N}$ is surjective and defined in the same way. The first row is exact since $A^{s}$ is projective. $A^{t_{1} s}$ is the free module of vector columns of size $t_{1} s$ obtained by concatenating the columns of matrices of $M_{t_{1} s}(A)$. $A^{t s}$ is defined in the same way, thus the vertical arrows are natural isomorphisms. The left vertical compose isomorphism is noted by $\Phi_{s, t_{1}}$ and the right compose isomorphism is noted by $\Phi_{s, t} . d_{N}^{*}$ is induced by $d_{N}$ and the vertical isomorphisms, $d_{N}^{*}=\Phi_{s, t} \circ d_{N} \circ \Phi_{s, t_{1}}^{-1}$. Then, the following diagram 
is commutative:

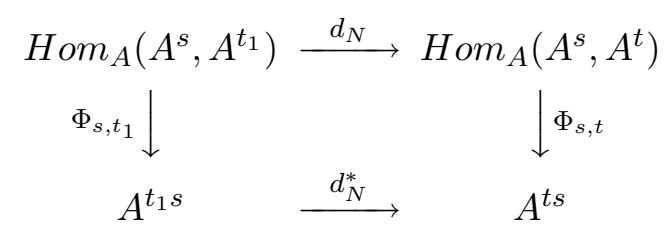

We can explicit how acts the homomorphism $d_{N}^{*}$, in fact, if

$$
\boldsymbol{a}=\left(a_{11}, \ldots, a_{t_{1} 1}, \ldots, a_{1 s}, \ldots, a_{t_{1} s}\right) \in A^{t_{1} s}
$$

and $\Delta_{N}=\left[\Delta_{i j}\right]$ is the matrix of $\delta_{N}$ in the canonical bases of $A^{t_{1}}$ and $A^{t}$, then

$$
d_{N}^{*}(\boldsymbol{a})=\left(\sum_{k=1}^{t_{1}} \Delta_{1 k} a_{k 1}, \ldots, \sum_{k=1}^{t_{1}} \Delta_{t k} a_{k 1}, \ldots, \sum_{k=1}^{t_{1}} \Delta_{1 k} a_{k s}, \ldots, \sum_{k=1}^{t_{1}} \Delta_{t k} a_{k s}\right)
$$

Thus, in the canonical bases of $A^{t_{1} s}$ and $A^{t s}$ the matrix of $d_{N}^{*}$ is

$$
I_{s} \otimes \Delta_{N}
$$

where $I_{s}$ is the identical matrix of size $s \times$ and $\otimes$ means tensor product. Thus, $d_{N}^{*}(\boldsymbol{a})=\left(I_{s} \otimes \Delta_{N}\right) \boldsymbol{a}^{T}$. From the exact sequence (3.4) we get that

$$
\operatorname{Hom}_{A}\left(A^{s}, A^{t} / \operatorname{Syz}(N)\right) \cong \operatorname{Hom}_{A}\left(A^{s}, A^{t}\right) / \operatorname{Im}\left(d_{N}\right),
$$

but

$$
\operatorname{Im}\left(d_{N}\right) \cong \operatorname{Im}\left(d_{N}^{*}\right)=<I_{s} \otimes \Delta_{N}>,
$$

where $\left\langle I_{s} \otimes \Delta_{N}>\right.$ is the module generated by the columns of $I_{s} \otimes \Delta_{N}$.

Hence a presentation of $\operatorname{Hom}_{A}\left(A^{s}, A^{t} / S y z(N)\right)$ is

$$
\operatorname{Hom}_{A}\left(A^{s}, A^{t} / \operatorname{Syz}(N)\right) \cong A^{t s} /<I_{s} \otimes \Delta_{N}>.
$$

This isomorphism is defined as follow. Let $f \in \operatorname{Hom}_{A}\left(A^{s}, A^{t} / \operatorname{Syz}(N)\right)$ and $f\left(\boldsymbol{e}_{i}\right)=\overline{\left(f_{1 i}, \ldots, f_{t i}\right)}, 1 \leq i \leq s$, then

$$
\operatorname{Hom}_{A}\left(A^{s}, A^{t} / \operatorname{Syz}(N)\right) \stackrel{\theta_{s, t}}{\longrightarrow} A^{t s} /<I_{s} \otimes \Delta_{N}>
$$

is defined by

$$
\theta_{s, t}(f)=\overline{\left(f_{11}, \ldots, f_{t 1}, \ldots, f_{1 s}, \ldots, f_{t s}\right)} .
$$

We observe that $\theta_{s, t}$ is a surjective homomorphism. We have to prove that $\theta_{s, t}$ is injective. If $\theta_{s, t}(f)=\overline{\mathbf{0}}$, then $\left(f_{11}, \ldots, f_{t 1}, \ldots, f_{1 s}, \ldots, f_{t s}\right) \in$ $<I_{s} \otimes \Delta_{N}>$ and consequently

$$
\left(f_{11}, \ldots, f_{t 1}, \ldots, f_{1 s}, \ldots, f_{t s}\right)=d_{N}^{*}(\boldsymbol{a})
$$


for some $\boldsymbol{a}=\left(a_{11}, \ldots, a_{t_{1} 1}, \ldots, a_{1 s}, \ldots, a_{t_{1} s}\right)$. Thus,

$$
\begin{aligned}
\left(f_{11}, \ldots, f_{t 1}, \ldots, f_{1 s}, \ldots, f_{t s}\right) & =\Phi_{s, t} \circ d_{N} \circ \Phi_{s, t_{1}}^{-1}(\boldsymbol{a}) \\
& =\Phi_{s, t} \circ d_{N}(a) \\
& =\Phi_{s, t}\left(\delta_{N} \circ a\right),
\end{aligned}
$$

where $a \in \operatorname{Hom}_{A}\left(A^{s}, A^{t_{1}}\right)$ is defined by $a\left(\boldsymbol{e}_{i}\right)=\left(a_{1 i}, \ldots, a_{t_{1} i}\right), 1 \leq i \leq$ $s$. But for each $1 \leq i \leq s,\left(\delta_{N} \circ a\right)\left(\boldsymbol{e}_{i}\right) \in<\Delta_{N}>=\operatorname{Syz}(N)$, hence $\overline{\left(f_{1 i}, \ldots, f_{t i}\right)}=\overline{\mathbf{0}}$ for each $1 \leq i \leq s$. This means that $f=0$.

In the same way, but using $\operatorname{Hom}_{A}\left(A^{s_{1}}\right.$, ), we obtain

$$
\operatorname{Hom}_{A}\left(A^{s_{1}}, A^{t} / \operatorname{Syz}(N)\right) \cong A^{t s_{1}} /<I_{s_{1}} \otimes \Delta_{N}>,
$$

and the explicit isomorphism

$$
\operatorname{Hom}_{A}\left(A^{s_{1}}, A^{t} / \operatorname{Syz}(N)\right) \stackrel{\theta_{s_{1}, t}}{\longrightarrow} A^{t s_{1}} /<I_{s_{1}} \otimes \Delta_{N}>
$$

is defined by

$$
\theta_{s_{1}, t}(h)=\overline{\left(h_{11}, \ldots, h_{t 1}, \ldots, h_{1 s_{1}}, \ldots, h_{t s_{1}}\right)},
$$

where $h \in \operatorname{Hom}_{A}\left(A^{s_{1}}, A^{t} / \operatorname{Syz}(N)\right)$.

In the example we have

$$
<I_{2} \otimes \Delta_{N}>=\left[\begin{array}{cccc}
5 & 2 x+9 y & 0 & 0 \\
0 & 8 x & 0 & 0 \\
5 & y & 0 & 0 \\
0 & 0 & 5 & 2 x+9 y \\
0 & 0 & 0 & 8 x \\
0 & 0 & 5 & y
\end{array}\right],<I_{1} \otimes \Delta_{N}>=\left[\begin{array}{cc}
5 & 2 x+9 y \\
0 & 8 x \\
5 & y
\end{array}\right]
$$

and hence

$$
\begin{gathered}
\operatorname{Hom}_{A}\left(A^{2}, A^{3} /<(5,0,5),(2 x+9 y, 8 x, y)>\right) \cong A^{6} /<I_{2} \otimes \Delta_{N}>, \\
\operatorname{Hom}_{A}\left(A, A^{3} /<(5,0,5),(2 x+9 y, 8 x, y)>\right) \cong A^{3} /<I_{1} \otimes \Delta_{N}>.
\end{gathered}
$$

Step 4. Computing the matrix $U$. As in the previous step, the homomorphism $\delta_{M}$ in the sequence (3.1) induces the natural homomorphism $d_{M}$, and this one induces the homomorphism $d_{M}^{*}$ defined by $d_{M}$ and the natural vertical isomorphisms of the following commutative diagram

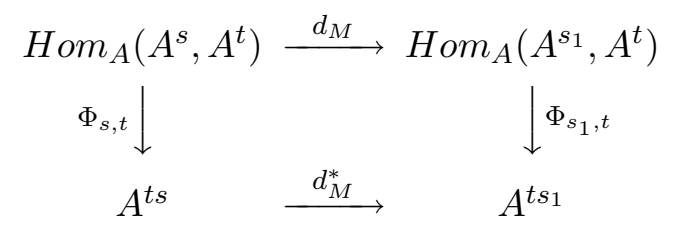


Since $\operatorname{Hom}_{A}\left(, A^{t}\right)$ inverts the sense of arrows, in the canonical bases the matrix of $d_{M}^{*}$ is

$$
\Delta_{M}^{T} \otimes I_{t}=\left(\Delta_{M} \otimes I_{t}\right)^{T}
$$

where $\Delta_{M}^{T}$ is the transpose of the matrix $\Delta_{M}$. In the example,

$$
\left(\Delta_{M} \otimes I_{3}\right)^{T}=\left[\begin{array}{cccccc}
5 y & 0 & 0 & 5 x & 0 & 0 \\
0 & 5 y & 0 & 0 & 5 x & 0 \\
0 & 0 & 5 y & 0 & 0 & 5 x
\end{array}\right] .
$$

As in the equation (3.5), we can explicit the homomorphism $d_{M}^{*}$. Let $\boldsymbol{u}=$ $\left(u_{11}, \ldots, u_{t 1}, \ldots, u_{1 s}, \ldots, u_{t s}\right)$, then $d_{M}^{*}(\boldsymbol{u})=\left(\Delta_{M} \otimes I_{t}\right)^{T} \boldsymbol{u}^{T}$, and hence

$$
d_{M}^{*}(\boldsymbol{u})=\left(\sum_{k=1}^{s} u_{1 k} \delta_{k 1}, \ldots, \sum_{k=1}^{s} u_{t k} \delta_{k 1}, \ldots, \sum_{k=1}^{s} u_{1 k} \delta_{k s_{1}}, \ldots, \sum_{k=1}^{s} u_{t k} \delta_{k s_{1}}\right)
$$

where $\Delta_{M}=\left[\delta_{v z}\right]$.

We observe that $\left.d_{M}^{*}\left(<I_{s} \otimes \Delta_{N}\right\rangle\right) \subseteq<I_{s_{1}} \otimes \Delta_{N}>$. In fact, it is enough to prove that $d_{M}^{*}(\boldsymbol{u}) \in<I_{s_{1}} \otimes \Delta_{N}>$, where $\boldsymbol{u}$ is any column of $I_{s} \otimes \Delta_{N}$. But the form of $\boldsymbol{u}$ is

$$
\boldsymbol{u}=\left(0, \ldots, 0, \ldots, \Delta_{1 j}, \ldots, \Delta_{t j}, \ldots, 0, \ldots, 0\right)
$$

where $\left(\Delta_{1 j}, \ldots, \Delta_{t j}\right)$ is the $j$-column of $\Delta_{N}, 1 \leq j \leq t_{1}$. We note that $\left(\Delta_{1 j}, \ldots, \Delta_{t j}\right)$ can be located in $s$ different places within $\boldsymbol{u}$, so we suppose that $\left(\Delta_{1 j}, \ldots, \Delta_{t j}\right)$ is located in the $l$-position within $\boldsymbol{u}, 1 \leq l \leq s$. Thus,

$$
d_{M}^{*}(\boldsymbol{u})=\left(\Delta_{M} \otimes I_{t}\right)^{T} \boldsymbol{u}^{T}=\left[\begin{array}{c}
\delta_{l 1} \Delta_{1 j} \\
\vdots \\
\delta_{l 1} \Delta_{t j} \\
\vdots \\
\delta_{l s_{1}} \Delta_{1 j} \\
\vdots \\
\delta_{l s_{1}} \Delta_{t j}
\end{array}\right]=\delta_{l 1}\left[\begin{array}{c}
\Delta_{1 j} \\
\vdots \\
\Delta_{t j} \\
0 \\
\vdots \\
0
\end{array}\right]+\cdots+\delta_{l s_{1}}\left[\begin{array}{c}
0 \\
\vdots \\
0 \\
\Delta_{1 j} \\
\vdots \\
\Delta_{t j}
\end{array}\right]
$$

i.e., $d_{M}^{*}(\boldsymbol{u}) \in<I_{s_{1}} \otimes \Delta_{N}>$. 
The homomorphism $d_{M}^{*}$ induces the homomorphism $\overline{d_{M}^{*}}$ and we have the commutative diagram

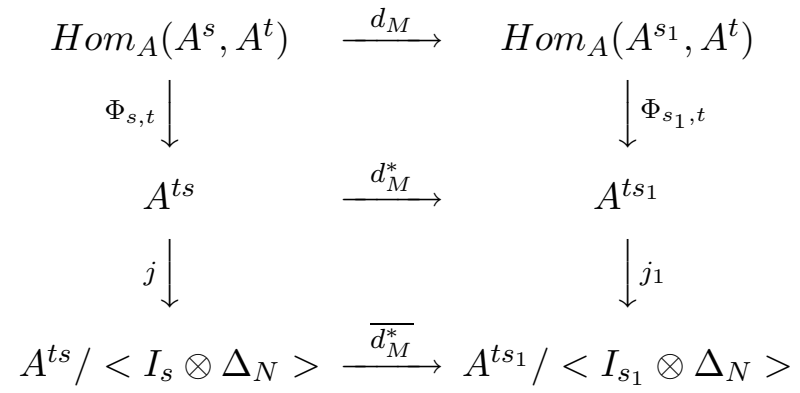

where $j$ and $j_{1}$ are the canonical homomorphisms. We define the matrix $U$ where its columns are the generators of $\operatorname{ker}\left(j_{1} \circ d_{M}^{*}\right)$, i.e., $\langle U>=$ $\operatorname{ker}\left(j_{1} \circ d_{M}^{*}\right)$. We observe that the matrix of homomorphism $j_{1} \circ d_{M}^{*}$ coincides with the matrix of homomorphism $d_{M}^{*}$, then by the Theorem 8 ,

columns of $U=$ first st coordinates of generators of

$$
\left.\operatorname{Syz}\left(\left[\left(\Delta_{M} \otimes I_{t}\right)^{T} \mid I_{s_{1}} \otimes \Delta_{N}\right)\right]\right)
$$

(the enhanced matrix obtained from $A$ adding the columns of $B$ is denoted by $[A \mid B])$. For our illustrative example, we have computed $\operatorname{Syz}\left(\left[\left(\Delta_{M} \otimes\right.\right.\right.$ $\left.\left.\left.\left.I_{3}\right)^{T} \mid I_{1} \otimes \Delta_{N}\right)\right]\right)$ using Theorem 4. Additionally, we computed a minimal Gröbner basis for $\left.\operatorname{Syz}\left(\left[\left(\Delta_{M} \otimes I_{3}\right)^{T} \mid I_{1} \otimes \Delta_{N}\right)\right]\right)$ and then we selected the first six coordinates of elements of this basis, the result of these computations was

$$
U=\left[\begin{array}{cccccccc}
1 & 0 & 0 & 0 & 0 & 0 & 0 & 0 \\
0 & x & 2 & 0 & 0 & 0 & 0 & 0 \\
1 & 0 & 0 & x & 2 & 0 & 0 & 0 \\
0 & 0 & 0 & y & 0 & 1 & 0 & 0 \\
0 & 9 y & 0 & 0 & 0 & 0 & 2 & 0 \\
0 & 0 & 0 & 0 & 0 & 1 & 0 & 2
\end{array}\right]
$$

Step 5. Presentation of $\operatorname{Hom}_{A}\left(A^{s} / \operatorname{Syz}(M), A^{t} / \operatorname{Syz}(N)\right)$. From the above results we have the following commutative diagram

$$
\begin{array}{ccc}
\operatorname{Hom}_{A}\left(A^{s}, A^{t} / \operatorname{Syz}(N)\right) & d & \operatorname{Hom}_{A}\left(A^{s_{1}}, A^{t} / \operatorname{Syz}(N)\right) \\
\theta_{s, t} \downarrow & & \downarrow_{s_{1}, t} \\
A^{t s} /<I_{s} \otimes \Delta_{N}> & \stackrel{\frac{}{d_{M}^{*}}}{\longrightarrow} & A^{t s_{1}} /<I_{s_{1}} \otimes \Delta_{N}>.
\end{array}
$$

In fact, let $f \in \operatorname{Hom}_{A}\left(A^{s}, A^{t} / \operatorname{Syz}(N)\right)$ with matrix $F=\left[f_{j i}\right]$ given by $f\left(\boldsymbol{e}_{i}\right)=\overline{\left(f_{1 i}, \ldots, f_{t i}\right)}, 1 \leq i \leq s$. Then, 


$$
d(f)=f \circ \delta_{M}=h \in \operatorname{Hom}_{A}\left(A^{s_{1}}, A^{t} / \operatorname{Syz}(N)\right),
$$

and hence,

$$
\frac{\theta_{s_{1}, t}(h)=}{\left(\sum_{k=1}^{s} f_{1 k} \delta_{k 1}, \ldots, \sum_{k=1}^{s} f_{t k} \delta_{k 1}, \ldots, \sum_{k=1}^{s} f_{1 k} \delta_{k s_{1}}, \ldots, \sum_{k=1}^{s} f_{t k} \delta_{k s_{1}}\right)} .
$$

On the other hand,

$$
\begin{aligned}
\overline{d_{M}^{*}}\left(\theta_{s, t}(f)\right) & =\overline{d_{M}^{*}}\left(\overline{\left(f_{11}, \ldots, f_{t 1}, \ldots, f_{1 s}, \ldots, f_{t s}\right)}\right) \\
& =\overline{d_{M}^{*}\left(\left(f_{11}, \ldots, f_{t 1}, \ldots, f_{1 s}, \ldots, f_{t s}\right)\right.} \\
& =\frac{\left(\Delta_{M}^{T} \otimes I_{t}\right)\left(f_{11}, \ldots, f_{t 1}, \ldots, f_{1 s}, \ldots, f_{t s}\right)}{s} \\
& =\left(\sum_{k=1}^{s} f_{1 k} \delta_{k 1}, \ldots, \sum_{k=1}^{s} f_{t k} \delta_{k 1}, \ldots, \sum_{k=1}^{s} f_{1 k} \delta_{k s_{1}}, \ldots, \sum_{k=1}^{s} f_{t k} \delta_{k s_{1}}\right) .
\end{aligned}
$$

Hence, $\operatorname{ker}(d) \cong \operatorname{ker}\left(\overline{d_{M}^{*}}\right)$, and from $(3.3)$, a presentation of $\operatorname{ker}\left(\overline{d_{M}^{*}}\right)$ gives a presentation of $\operatorname{Hom}_{A}\left(A^{s} / \operatorname{Syz}(M), A^{t} / \operatorname{Syz}(N)\right)$. Let $\mathbf{u} \in A^{t s}$, then

$$
\begin{gathered}
\overline{\mathbf{u}} \in \operatorname{ker}\left(\overline{d_{M}^{*}}\right) \Longleftrightarrow \overline{d_{M}^{*}}(\overline{\mathbf{u}})=\overline{\mathbf{0}} \Longleftrightarrow d_{M}^{*}(\mathbf{u}) \in<I_{s_{1}} \otimes \Delta_{N}>\Longleftrightarrow \\
\left(\Delta_{M} \otimes I_{t}\right)^{T} \boldsymbol{u}^{T} \in<I_{s_{1}} \otimes \Delta_{N}>\Longleftrightarrow
\end{gathered}
$$

the first st coordiantes of $\mathbf{u}$ belong to $\left.\operatorname{Syz}\left(\left[\left(\Delta_{M} \otimes I_{t}\right)^{T} \mid I_{s_{1}} \otimes \Delta_{N}\right)\right]\right) \Longleftrightarrow$

$$
\overline{\mathbf{u}} \in<U>/<I_{s} \otimes \Delta_{N}>
$$

where $\langle U\rangle$ is the column module of matrix $U$. Thus, we have proved that $\operatorname{ker}\left(\overline{d_{M}^{*}}\right)=\left\langle U>/<I_{s} \otimes \Delta_{N}>\right.$, and we get the following theorem.

Theorem 15. With the notation above,

$$
\operatorname{Hom}_{A}(M, N) \cong<U>/<I_{s} \otimes \Delta_{N}>,
$$

and presentation of $\langle U\rangle /\left\langle I_{s} \otimes \Delta_{N}\right\rangle$ is a presentation for $\operatorname{Hom}_{A}(M, N)$.

In the example we have $\operatorname{Hom}_{A}(M, N) \cong A^{8} / K$, where $K$ is the module generated by the first 8 entries of generators of $\operatorname{Syz}\left(\left[U \mid I_{2} \otimes \Delta_{N}\right]\right.$ ) (see the Theorem 7). We computed a minimal Gröbner basis for the syzygy of the matrix $\left[U \mid I_{2} \otimes \Delta_{N}\right]$ and then we selected the first eight entries of the elements of this basis. The result was

$$
\operatorname{Hom}_{A}(M, N) \cong A^{8} /<\boldsymbol{v}_{1}, \boldsymbol{v}_{2}, \mathbf{v}_{3}, \boldsymbol{v}_{4}, \boldsymbol{v}_{5}, \boldsymbol{v}_{6}, \boldsymbol{v}_{7}, \boldsymbol{v}_{8}, \boldsymbol{v}_{9}>
$$


where

$$
\begin{aligned}
& \boldsymbol{v}_{1}=<5,0,0,0,0,0,0,0> \\
& \boldsymbol{v}_{2}=<8 x+y, 0,6 x, 0, x+4 y, 0,0,0> \\
& \boldsymbol{v}_{3}=<0,0,5,0,0,0,0,0> \\
& \boldsymbol{v}_{4}=<0,8, x, 0,0,0,4 y, 0> \\
& \boldsymbol{v}_{5}=<0,0,0,0,5,0,0,0> \\
& \boldsymbol{v}_{6}=<0,0,0,8, x, 2 y, 0,0> \\
& \boldsymbol{v}_{7}=<0,0,0,0,0,0,5,0> \\
& \boldsymbol{v}_{8}=<0,0,0,0,0,0,0,5> \\
& \boldsymbol{v}_{9}=<0,0,0,0,0,4 x+8 y, 8 x, y>.
\end{aligned}
$$

Step 6. We conclude this section computing an explicit set of generators for $\operatorname{Hom}_{A}(M, N)$ in our illustrative example. We know that

$\operatorname{Hom}_{A}(M, N) \cong$

$$
\operatorname{Hom}_{A}\left(A^{2} /<(5 y, 5 x)>, A^{3} /<(5,0,5),(2 x+9 y, 8 x, y)>\right) \text {. }
$$

Each element $\phi \in \operatorname{Hom}_{A}(M, N)$ can be represented by an unique element $\overline{\boldsymbol{a}} \in A^{8} /\left\langle\boldsymbol{v}_{1}, \ldots, \boldsymbol{v}_{9}>\right.$ with $\boldsymbol{a}=a_{1} \boldsymbol{e}_{1}+\cdots+a_{8} \boldsymbol{e}_{8} \in A^{8}$. We consider the column $i$ of $U, U \boldsymbol{e}_{i}, 1 \leq i \leq 8$, the six entries of this column could be disposed into a $3 \times 2$ matrix, denoted by $U_{i}$, taking the first three entries as column one and the next three entries as column two. Thus, $U_{i}$ represents an $A$-homomorphism defined by

$$
\begin{aligned}
A^{2} \stackrel{U_{i}}{\longrightarrow} A^{3} \\
\boldsymbol{z} \mapsto U_{i} \boldsymbol{z}
\end{aligned}
$$

and it induces an $A$-homomorphism also denoted by $U_{i}$

$$
\begin{aligned}
A^{2} / K_{M} \stackrel{U_{i}}{\longrightarrow} A^{3} / S y z(N) \\
\overline{\boldsymbol{z}} \mapsto \overline{U_{i} \boldsymbol{z}} .
\end{aligned}
$$

It is easy to verify that each $U_{i}$ is well defined, i.e. $U_{i}\left(K_{M}\right) \subseteq K_{N}, 1 \leq i \leq$ 8. The eight matrices are

$$
\begin{gathered}
U_{1}=\left[\begin{array}{ll}
1 & 0 \\
0 & 0 \\
1 & 0
\end{array}\right], U_{2}=\left[\begin{array}{cc}
0 & 0 \\
x & 9 y \\
0 & 0
\end{array}\right] U_{3}=\left[\begin{array}{ll}
0 & 0 \\
2 & 0 \\
0 & 0
\end{array}\right], U_{4}=\left[\begin{array}{ll}
0 & y \\
0 & 0 \\
x & 0
\end{array}\right] \\
U_{5}=\left[\begin{array}{ll}
0 & 0 \\
0 & 0 \\
2 & 0
\end{array}\right] U_{6}=\left[\begin{array}{ll}
0 & 1 \\
0 & 0 \\
0 & 1
\end{array}\right] U_{7}=\left[\begin{array}{ll}
0 & 0 \\
0 & 2 \\
0 & 0
\end{array}\right] U_{8}=\left[\begin{array}{ll}
0 & 0 \\
0 & 0 \\
0 & 2
\end{array}\right] .
\end{gathered}
$$


The isomorphisms

$$
\begin{aligned}
A^{2} / K_{M} & \cong M & A^{3} / K_{N} & \cong N \\
\overline{\boldsymbol{e}_{i}} & \mapsto \boldsymbol{f}_{i} & \overline{\boldsymbol{e}_{j}^{\prime}} & \mapsto \boldsymbol{g}_{j}
\end{aligned}
$$

describe the eight homomorphisms of $\operatorname{Hom}_{A}(M, N)$,

$$
\begin{array}{lcc}
M \stackrel{\phi_{1}}{\longrightarrow} N & \\
\boldsymbol{f}_{1} \mapsto \overline{\boldsymbol{e}_{1}} \mapsto \overline{U_{1} \boldsymbol{e}_{1}} & =\overline{\boldsymbol{e}_{1}^{\prime}}+\overline{\boldsymbol{e}_{3}^{\prime}}=\boldsymbol{g}_{1}+\boldsymbol{g}_{3} \\
\boldsymbol{f}_{2} \mapsto \overline{\boldsymbol{e}_{2}} \mapsto \overline{U_{1} \boldsymbol{e}_{2}} & =\mathbf{0} & \\
M \stackrel{\phi_{2}}{\longrightarrow} N & M \stackrel{\phi_{3}}{\longrightarrow} N & M \stackrel{\phi_{4}}{\longrightarrow} N \\
\boldsymbol{f}_{1} \mapsto x \boldsymbol{g}_{2} & \boldsymbol{f}_{1} \mapsto 2 \boldsymbol{g}_{2} & \boldsymbol{f}_{1} \mapsto x \boldsymbol{g}_{3} \\
\boldsymbol{f}_{2} \mapsto 9 y \boldsymbol{g}_{2} & \boldsymbol{f}_{2} \mapsto \mathbf{0} & \boldsymbol{f}_{2} \mapsto y \boldsymbol{g}_{1} \\
& M \stackrel{\phi_{6}}{\longrightarrow} N & \\
M \stackrel{\phi_{5}}{\longrightarrow} N & \mathbf{f}_{1} \mapsto \mathbf{0} & \\
\boldsymbol{f}_{1} \mapsto 2 \boldsymbol{g}_{3} & \boldsymbol{f}_{2} \mapsto \boldsymbol{g}_{1}+\boldsymbol{g}_{3} & \boldsymbol{f}_{1} \mapsto \mathbf{0} \\
\boldsymbol{f}_{2} \mapsto \mathbf{0} & & \boldsymbol{f}_{2} \mapsto 2 \boldsymbol{g}_{2} \\
& M \stackrel{\phi_{8}}{\longrightarrow} N & \\
& \boldsymbol{f}_{1} \mapsto \mathbf{0} & \\
& \boldsymbol{f}_{2} \mapsto 2 \boldsymbol{g}_{3} &
\end{array}
$$

3.2. Computation of $M \otimes N$. For $M=<\boldsymbol{f}_{1}, \ldots, \boldsymbol{f}_{s}>\subseteq A^{m}$ and $N=<$ $\boldsymbol{g}_{1}, \ldots, \boldsymbol{g}_{t}>\subseteq A^{l}$ we now compute a presentation of $M \otimes N$. This computation has been also considered in [8] using exact sequences (see [8], Corollary 2.7.8). Our presentation is given as a quotient module of $M \otimes N$ and showing an explicit set of generators for $\operatorname{Syz}(M \otimes N)$. We start with a preliminary proposition ( see [8], Proposition 2.7.10).

Proposition 16. Let $S$ be an arbitrary commutative ring and $M, N \bmod$ ules over $S$. Let $\boldsymbol{m}_{j} \in M, \boldsymbol{g}_{j} \in N, 1 \leq j \leq t$ such that $N=<\boldsymbol{g}_{1}, \ldots, \boldsymbol{g}_{t}>$. Then, $\boldsymbol{m}_{1} \otimes \boldsymbol{g}_{1}+\cdots+\boldsymbol{m}_{t} \otimes \boldsymbol{g}_{t}=\mathbf{0}$ if and only if there exist elements $\boldsymbol{m}_{v}^{\prime} \in M$ and $h_{j v} \in S$, such that $\boldsymbol{m}_{j}=\sum_{v=1}^{r} h_{j v} \boldsymbol{m}_{v}^{\prime}$ and $\sum_{j=1}^{t} h_{j v} \boldsymbol{g}_{j}=\mathbf{0}$ for each $1 \leq v \leq r, 1 \leq j \leq t$. In a matrix notation, 


$$
\left[\boldsymbol{m}_{1} \cdots \boldsymbol{m}_{t}\right]=\left[\boldsymbol{m}_{1}^{\prime} \cdots \boldsymbol{m}_{r}^{\prime}\right] H^{T}, H^{T}\left[\boldsymbol{g}_{1} \cdots \boldsymbol{g}_{t}\right]=0,
$$

where $H=\left[h_{j v}\right]$ and the module expanded by the columns of $H$ is contained in $\operatorname{Syz}(N)$.

Theorem 17. Let $M$ and $N$ be submodules as above. Then,

$$
M \otimes N \cong A^{s t} / \operatorname{Syz}(M \otimes N)
$$

where

$$
\operatorname{Syz}(M \otimes N)=<\left[\operatorname{Syz}(M) \otimes I_{t} \mid I_{s} \otimes S y z(N)\right]>.
$$

Proof. It is clear that

$$
M \otimes N=<\boldsymbol{f}_{1} \otimes \boldsymbol{g}_{1}, \ldots, \boldsymbol{f}_{1} \otimes \boldsymbol{g}_{t}, \ldots, \boldsymbol{f}_{s} \otimes \boldsymbol{g}_{1}, \ldots, \boldsymbol{f}_{s} \otimes \boldsymbol{g}_{t}>.
$$

Let $\operatorname{Syz}(M)=<\boldsymbol{f}_{1}^{\prime}, \ldots, \boldsymbol{f}_{r}^{\prime}>$ and $S y z(N)=<\boldsymbol{g}_{1}^{\prime}, \ldots, \boldsymbol{g}_{p}^{\prime}>$, with

$$
\boldsymbol{f}_{1}^{\prime}=\left(f_{11}, \ldots, f_{s 1}\right), \ldots, \boldsymbol{f}_{r}^{\prime}=\left(f_{1 r}, \ldots, f_{s r}\right)
$$

and

$$
\boldsymbol{g}_{1}^{\prime}=\left(g_{11}, \ldots, g_{t 1}\right), \ldots, \boldsymbol{g}_{p}^{\prime}=\left(g_{1 p}, \ldots, g_{t p}\right) .
$$

In a matrix notation,

$$
\operatorname{Syz}(M)=\left[\begin{array}{ccc}
f_{11} & \ldots & f_{1 r} \\
\vdots & \ldots & \vdots \\
f_{s 1} & \cdots & f_{s r}
\end{array}\right], \operatorname{Syz}(N)=\left[\begin{array}{ccc}
g_{11} & \ldots & g_{1 p} \\
\vdots & \ldots & \vdots \\
g_{t 1} & \cdots & g_{t p}
\end{array}\right] .
$$

Then,

$$
\begin{gathered}
f_{11} \boldsymbol{f}_{1}+\cdots+f_{s 1} \boldsymbol{f}_{s}=\mathbf{0} \\
\vdots \\
f_{1 r} \boldsymbol{f}_{1}+\cdots+f_{s r} \boldsymbol{f}_{s}=\mathbf{0}
\end{gathered}
$$

and

$$
\begin{gathered}
g_{11} \boldsymbol{g}_{1}+\cdots+g_{t 1} \boldsymbol{g}_{t}= \\
\vdots \\
g_{1 p} \boldsymbol{g}_{1}+\cdots+g_{t p} \boldsymbol{g}_{t}=\mathbf{0} .
\end{gathered}
$$

We note that any of the following $t r$ vectors has $s t$ entries and belongs to $\operatorname{Syz}(M \otimes N)$ 


$$
\begin{gathered}
\left(f_{11}, 0, \ldots, 0, \ldots, f_{s 1}, 0, \ldots, 0\right) \\
\vdots \\
\left(0,0, \ldots, f_{11}, \ldots, 0,0, \ldots, f_{s 1}\right) \\
\vdots \\
\left(f_{1 r}, 0, \ldots, 0, \ldots, f_{s r}, 0, \ldots, 0\right) \\
\vdots \\
\left(0,0, \ldots, f_{1 r}, \ldots, 0,0, \ldots, f_{s r}\right) .
\end{gathered}
$$

In the same way, any of the following $p s$ vectors has $s t$ entries and belongs to $\operatorname{Syz}(M \otimes N)$

$$
\begin{gathered}
\left(g_{11}, \ldots, g_{t 1}, \ldots, 0, \ldots, 0\right) \\
\ddots \\
\left(0, \ldots, 0, \ldots, g_{11}, \ldots, g_{t 1}\right) \\
\vdots \\
\left(g_{1 p}, \ldots, g_{t p}, \ldots, 0, \ldots, 0\right) \\
\ddots \\
\left(0, \ldots, 0, \ldots, g_{1 p}, \ldots, g_{t p}\right) .
\end{gathered}
$$

We can dispose these $t r+p s$ vectors by columns in a matrix $[C \mid B]$ of size $s t \times(t r+p s)$, where

$$
\begin{gathered}
C=\left[\begin{array}{ccccccc}
f_{11} & \ldots & 0 & \ldots & f_{1 r} & \ldots & 0 \\
& \ddots & & \ldots & & \ddots & \\
0 & \ldots & f_{11} & \ldots & 0 & \ldots & f_{1 r} \\
& \vdots & & \ldots & & \vdots & \\
f_{s 1} & \ldots & 0 & \ldots & f_{s r} & \ldots & 0 \\
& \ddots & & \ldots & & \ddots & \\
0 & \ldots & f_{s 1} & \ldots & 0 & \ldots & f_{s r}
\end{array}\right]=\operatorname{Syz}(M) \otimes I_{t} \\
B=\left[\begin{array}{ccccccc}
g_{11} & \ldots & 0 & \ldots & g_{1 p} & \ldots & 0 \\
\vdots & \vdots & \vdots & \ldots & \vdots & \vdots & \vdots \\
g_{t 1} & \ldots & 0 & \ldots & g_{t p} & \ldots & 0 \\
\vdots & \ddots & \vdots & \ldots & \vdots & \ddots & \vdots \\
0 & \ldots & g_{11} & \ldots & 0 & \ldots & g_{1 p} \\
\vdots & \vdots & \vdots & \ldots & \vdots & \vdots & \vdots \\
0 & \ldots & g_{t 1} & \ldots & 0 & \ldots & g_{t p}
\end{array}\right]
\end{gathered}
$$

São Paulo J.Math.Sci. 3, 1 (2009), 25-59 
But $B$ can be changed by

$$
\left[\begin{array}{ccccccc}
g_{11} & \ldots & g_{1 p} & \ldots & 0 & \ldots & 0 \\
\vdots & \vdots & \vdots & \ldots & \vdots & \vdots & \vdots \\
g_{t 1} & \ldots & g_{t p} & \ldots & 0 & \ldots & 0 \\
\vdots & \vdots & \vdots & \ddots & \vdots & \vdots & \vdots \\
0 & \ldots & 0 & \ldots & g_{11} & \ldots & g_{1 p} \\
\vdots & \vdots & \vdots & \ldots & \vdots & \vdots & \vdots \\
0 & \ldots & 0 & \ldots & g_{t 1} & \ldots & g_{t p}
\end{array}\right]=I_{s} \otimes \operatorname{Syz}(N) .
$$

Thus, we have proved that $<\left[\operatorname{Syz}(M) \otimes I_{t} \mid I_{s} \otimes S y z(N)\right]>\subseteq S y z(M \otimes$ $N)$.

Now we assume that $\boldsymbol{h}=\left(h_{11}, \ldots, h_{1 t}, \ldots, h_{s 1}, \ldots, h_{s t}\right) \in \operatorname{Syz}(M \otimes N)$, then

$h_{11}\left(\boldsymbol{f}_{1} \otimes \boldsymbol{g}_{1}\right)+\cdots+h_{1 t}\left(\boldsymbol{f}_{1} \otimes \boldsymbol{g}_{t}\right)+\cdots+h_{s 1}\left(\boldsymbol{f}_{s} \otimes \boldsymbol{g}_{1}\right)+\cdots+h_{s t}\left(\boldsymbol{f}_{s} \otimes \boldsymbol{g}_{t}\right)=\mathbf{0}$.

From this we get that

$$
\boldsymbol{m}_{1} \otimes \boldsymbol{g}_{1}+\cdots+\boldsymbol{m}_{t} \otimes \boldsymbol{g}_{t}=\mathbf{0},
$$

where

$$
\boldsymbol{m}_{j}=h_{1 j} \boldsymbol{f}_{1}+\cdots+h_{s j} \boldsymbol{f}_{s} \in M
$$

with $1 \leq j \leq t$. From the Proposition 16, there exist polynomials $a_{j v} \in A$ and vectors $\boldsymbol{m}_{v}^{\prime} \in M$ such that $\boldsymbol{m}_{j}=\sum_{v=1}^{r} a_{j v} \boldsymbol{m}_{v}^{\prime}$ and $\sum_{j=1}^{t} a_{j v} \boldsymbol{g}_{j}=\mathbf{0}$ for each $1 \leq v \leq r$. This means that $\left(a_{1 v}, \ldots, a_{t v}\right) \in \operatorname{Syz}(N)$ for each $1 \leq v \leq$ $r$. Since $\boldsymbol{m}_{v}^{\prime} \in M$ there exist $q_{u v} \in A$ such that $\boldsymbol{m}_{v}^{\prime}=q_{1 v} \boldsymbol{f}_{1}+\cdots+q_{s v} \boldsymbol{f}_{s}$, and then

$$
\begin{aligned}
\sum_{i=1}^{s} h_{i 1} \boldsymbol{f}_{i} & =a_{11}\left(q_{11} \boldsymbol{f}_{1}+\cdots+q_{s 1} \boldsymbol{f}_{s}\right)+\cdots+a_{1 r}\left(q_{1 r} \boldsymbol{f}_{1}+\cdots+q_{s r} \boldsymbol{f}_{s}\right) \\
& \vdots \\
\sum_{i=1}^{s} h_{i t} \boldsymbol{f}_{i} & =a_{t 1}\left(q_{11} \boldsymbol{f}_{1}+\cdots+q_{s 1} \boldsymbol{f}_{s}\right)+\cdots+a_{t r}\left(q_{1 r} \boldsymbol{f}_{1}+\cdots+q_{s r} \boldsymbol{f}_{s}\right) .
\end{aligned}
$$

From this we get that

$$
\begin{aligned}
& \sum_{i=1}^{s}\left(h_{i 1}-\left(a_{11} q_{i 1}+\cdots+a_{1 r} q_{i r}\right)\right) \boldsymbol{f}_{i}=\mathbf{0} \\
& \sum_{i=1}^{s}\left(h_{i t}-\left(a_{t 1} q_{i 1}+\cdots+a_{t r} q_{i r}\right)\right) \boldsymbol{f}_{i}=\mathbf{0}
\end{aligned}
$$

i.e., 


$$
\begin{gathered}
\left(h_{11}-\left(a_{11} q_{11}+\cdots+a_{1 r} q_{1 r}\right), \ldots, h_{s 1}-\left(a_{11} q_{s 1}+\cdots+a_{1 r} q_{s r}\right)\right) \in \operatorname{Syz}(M) \\
\vdots \\
\left(h_{1 t}-\left(a_{t 1} q_{11}+\cdots+a_{t r} q_{1 r}\right), \ldots, h_{s t}-\left(a_{t 1} q_{s 1}+\cdots+a_{t r} q_{s r}\right)\right) \in \operatorname{Syz}(M) .
\end{gathered}
$$

This implies that

$$
\begin{gathered}
\left(h_{11}, \ldots, h_{s 1}\right)-\left(a_{11} q_{11}+\cdots+a_{1 r} q_{1 r}, \ldots, a_{11} q_{s 1}+\cdots+a_{1 r} q_{s r}\right) \in \operatorname{Syz}(M) \\
\vdots \\
\left(h_{1 t}, \ldots, h_{s t}\right)-\left(a_{t 1} q_{11}+\cdots+a_{t r} q_{1 r}, \ldots, a_{t 1} q_{s 1}+\cdots+a_{t r} q_{s r}\right) \in \operatorname{Syz}(M) .
\end{gathered}
$$

Then,

$$
\begin{gathered}
\left(h_{i 1}\right)_{i=1}^{s}=\left(a_{11} q_{11}+\cdots+a_{1 r} q_{1 r}, \ldots, a_{11} q_{s 1}+\cdots+a_{1 r} q_{s r}\right)+\left(f_{11}, \ldots, f_{s 1}\right) \\
\vdots \\
\left(h_{i t}\right)_{i=1}^{s}=\left(a_{t 1} q_{11}+\cdots+a_{t r} q_{1 r}, \ldots, a_{t 1} q_{s 1}+\cdots+a_{t r} q_{s r}\right)+\left(f_{1 t}, \ldots, f_{s t}\right)
\end{gathered}
$$

with $\left(f_{11}, \ldots, f_{s 1}\right), \ldots,\left(f_{1 t}, \ldots, f_{s t}\right) \in S y z(M)$. From this we get

$$
\begin{gathered}
h_{11}=f_{11}+a_{11} q_{11}+\cdots+a_{1 r} q_{1 r} \\
\vdots \\
h_{1 t}=f_{1 t}+a_{t 1} q_{11}+\cdots+a_{t r} q_{1 r} \\
\vdots \\
h_{s 1}=f_{s 1}+a_{11} q_{s 1}+\cdots+a_{1 r} q_{s r} \\
\vdots \\
h_{s t}=f_{s t}+a_{t 1} q_{s 1}+\cdots+a_{t r} q_{s r},
\end{gathered}
$$

and hence $\boldsymbol{h}$ is a linear combination of columns of the following matrix

$$
\left[\operatorname{Syz}(M) \otimes I_{t} \mid D\right]
$$

where

$$
D=\left[\begin{array}{ccccccc}
a_{11} & \ldots & a_{1 r} & \ldots & 0 & \ldots & 0 \\
\vdots & \vdots & \vdots & \ldots & \vdots & \vdots & \vdots \\
a_{t 1} & \ldots & a_{t r} & \ldots & 0 & \ldots & 0 \\
\vdots & \vdots & \vdots & \ddots & \vdots & \vdots & \vdots \\
0 & \ldots & 0 & \ldots & a_{11} & \ldots & a_{1 r} \\
\vdots & \vdots & \vdots & \ldots & \vdots & \vdots & \vdots \\
0 & \ldots & 0 & \ldots & a_{t 1} & \ldots & a_{t r}
\end{array}\right]
$$


But $<D>\subseteq<I_{s} \otimes S y z(N)>$, and hence, $h \in<\left[\operatorname{Syz}(M) \otimes I_{t} \mid I_{s} \otimes\right.$ $S y z(N)]>$. This completes the proof of the proposition.

Example 18. Let $M$ and $N$ be submodules as in the Example 6, then

$$
\operatorname{Syz}(M \otimes N)=\left[\operatorname{Syz}(M) \otimes I_{3} \mid I_{2} \otimes S y z(N)\right],
$$

i.e.,

$$
\operatorname{Syz}(M \otimes N)=\left[\begin{array}{ccccccc}
5 y & 0 & 0 & 5 & 2 x+9 y & 0 & 0 \\
0 & 5 y & 0 & 0 & 8 x & 0 & 0 \\
0 & 0 & 5 y & 5 & y & 0 & 0 \\
5 x & 0 & 0 & 0 & 0 & 5 & 2 x+9 y \\
0 & 5 x & 0 & 0 & 0 & 0 & 8 x \\
0 & 0 & 5 x & 0 & 0 & 5 & y
\end{array}\right]
$$

3.3. Computing free resolutions. In this subsection we will compute free resolutions for submodules of $A^{m}$. Let $M$ be a submodule of $A^{m}$, we recall that a free resolution of $M$ is an exact sequence of free modules

$$
\cdots \stackrel{F_{r+2}}{\longrightarrow} A^{s_{r}} \stackrel{F_{r}}{\longrightarrow} A^{s_{r-1}} \stackrel{F_{r-1}}{\longrightarrow} \cdots \stackrel{F_{2}}{\longrightarrow} A^{s_{1}} \stackrel{F_{1}}{\longrightarrow} A^{s_{0}} \stackrel{F_{0}}{\longrightarrow} M \longrightarrow 0,
$$

with $s_{i} \geq 0$ for each $i \geq 0$. We assume that $A^{0}=0$. The following proposition describes a simple procedure for constructing a free resolution of $M$.

Theorem 19. Let $M=<f_{1}^{(0)}, \ldots, f_{s_{0}}^{(0)}>$ be a submodule of the free module $A^{m}$. Let $F_{0}$ be the matrix whose columns are $f_{1}^{(0)}, \ldots, f_{s_{0}}^{(0)}$, and for $i \geq 1$ let

$$
F_{i}=\operatorname{Syz}\left(F_{i-1}\right)=\left[\boldsymbol{f}_{1}^{(i)} \cdots \boldsymbol{f}_{s_{i}}^{(i)}\right] .
$$

Then,

$$
\ldots \stackrel{F_{r}+2}{\longrightarrow} A^{s_{r}} \stackrel{F_{r}}{\longrightarrow} A^{s_{r-1}} \stackrel{F_{r-1}}{\longrightarrow} \cdots \stackrel{F_{2}}{\longrightarrow} A^{s_{1}} \stackrel{F_{1}}{\longrightarrow} A^{s_{0}} \stackrel{F_{0}}{\longrightarrow} M \longrightarrow 0,
$$

is a free resolution of $M$, where

$$
F_{i} \boldsymbol{e}_{j_{i}}^{(i)}=\boldsymbol{f}_{j_{i}}^{(i)}
$$

and $\left\{\boldsymbol{e}_{j_{i}}^{(i)}\right\}_{1 \leq j_{i} \leq s_{i}}$ is the canonical basis of $A^{s_{i}}$.

Proof. Each homomorphism $F_{i}$ is represented by a matrix, and hence, a resolution of $M$ is described as a sequence of matrices $\left\{F_{i}\right\}_{i>0}$, where the columns of $F_{i}$ are the generators of $\operatorname{Syz}\left(F_{i-1}\right), i \geq 1$. The columns of $F_{0}$ are the generators of $M$. We note that $\operatorname{Im}\left(F_{i}\right)=\operatorname{Syz}\left(F_{i-1}\right)=\operatorname{ker}\left(F_{i-1}\right)$ for each $i \geq 1$, and moreover $F_{0}$ is a surjective homomorphism.

We can illustrate this procedure in the following example. 
Example 20. Let $M$ and $N$ be as in the Example 6, we will compute free resolutions for $M$ and $N$. According to the Proposition 19, we must compute the matrices $F_{i}, i \geq 0$, and for this, we will use the Theorem 4 .

We start with $M$, in this case we have

$$
\begin{gathered}
F_{0}=\left[\begin{array}{ll}
\boldsymbol{f}_{1} & \boldsymbol{f}_{2}
\end{array}\right]=\left[\begin{array}{cc}
3 x^{2} y+3 x & 7 x y^{2}+y \\
x y-2 y & y^{2}-4 x
\end{array}\right], \\
F_{1}=\operatorname{Syz}\left(F_{0}\right)=\left[\begin{array}{l}
5 y \\
5 x
\end{array}\right], F_{2}=\operatorname{Syz}\left(F_{1}\right)=[2], F_{3}=\operatorname{Syz}\left(F_{2}\right)=[5], \ldots
\end{gathered}
$$

hence, for $r \geq 1$

$$
F_{2 r}=[2], F_{2 r+1}=[5] .
$$

Thus, a free resolution for $M$ is

$$
\ldots \rightarrow A \stackrel{[5]}{\longrightarrow} A \stackrel{[2]}{\longrightarrow} A \stackrel{\left[\begin{array}{l}
5 y \\
5 x
\end{array}\right]}{\longrightarrow} A^{2} \stackrel{\left[\begin{array}{cc}
3 x^{2} y+3 x & 7 x y^{2}+y \\
x y-2 y & y^{2}-4 x
\end{array}\right]}{\longrightarrow} M \rightarrow 0 .
$$

For $N$ we have

$$
\begin{gathered}
G_{0}=\left[\begin{array}{lll}
\boldsymbol{g}_{1} & \boldsymbol{g}_{2} & \boldsymbol{g}_{3}
\end{array}\right]=\left[\begin{array}{ccc}
0 & y & 2 x \\
x & x & x
\end{array}\right], G_{1}=\operatorname{Syz}\left(G_{0}\right)=\left[\begin{array}{cc}
5 & 2 x+9 y \\
0 & 8 x \\
5 & y
\end{array}\right], \\
G_{2}=\operatorname{Syz}\left(G_{1}\right)=\left[\begin{array}{ll}
2 & y \\
0 & 5
\end{array}\right], G_{3}=\operatorname{Syz}\left(G_{2}\right)=\left[\begin{array}{ll}
5 & y \\
0 & 8
\end{array}\right], \\
G_{4}=\operatorname{Syz}\left(G_{3}\right)=\left[\begin{array}{ll}
2 & y \\
0 & 5
\end{array}\right], \ldots
\end{gathered}
$$

hence, for $r \geq 1$

$$
G_{2 r}=\left[\begin{array}{ll}
2 & y \\
0 & 5
\end{array}\right], G_{2 r+1}=\left[\begin{array}{ll}
5 & y \\
0 & 8
\end{array}\right] .
$$

Thus, a free resolution for $N$ is

$$
\begin{gathered}
\cdots \rightarrow A^{2} \stackrel{\left[\begin{array}{ll}
5 & y \\
0 & 8
\end{array}\right]}{\longrightarrow} A^{2} \stackrel{\left[\begin{array}{ll}
2 & y \\
0 & 5
\end{array}\right]}{\longrightarrow} A^{2} \\
A^{2} \stackrel{\left[\begin{array}{cc}
5 & 2 x+9 y \\
0 & 8 x \\
5 & y
\end{array}\right]}{\stackrel{[}{\longrightarrow}} A^{3} \stackrel{\left[\begin{array}{lll}
0 & y & 2 x \\
x & x & x
\end{array}\right]}{\longrightarrow} N \rightarrow 0
\end{gathered}
$$




\section{Computation of Ext and Tor}

Now we will present the main computations of this paper, the modules $\operatorname{Ext}_{A}^{r}(M)$ and $\operatorname{Tor}_{r}^{A}(M, N)$.

4.1. Computation of Ext. Using syzygies and the results of previous sections we now describe an easy procedure for computing the $A$-modules $\operatorname{Ext}_{A}^{r}(M, N)$ for $r \geq 0$, where $M=\left\langle\boldsymbol{f}_{1}, \ldots, \boldsymbol{f}_{s}\right\rangle \subseteq A^{m}$ and $N=<$ $\boldsymbol{g}_{1}, \ldots, \boldsymbol{g}_{t}>\subseteq A^{l}$. By computing we mean to find a presentation and a system of generators of $\operatorname{Ext}_{A}^{r}(M, N)$ (compare with [1], Section 3.10). For $r=0, \operatorname{Ext}_{A}^{0}(M, N)=\operatorname{Hom}_{A}(M, N)$ and the computation is given by the results of Section 3. So we assume that $r \geq 1$.

Presentation of $\operatorname{Ext}_{A}^{r}(M, N), r \geq 1$ :

Step 1. We compute presentations of $M$ and $N$,

$$
M \cong A^{s} / \operatorname{Syz}(M), N \cong A^{t} / \operatorname{Syz}(N),
$$

and we know how to compute $\operatorname{Syz}(M)$ and $\operatorname{Syz}(N)$.

Step 2. Using Theorem 19 we compute a free resolution of $A^{s} / \operatorname{Syz}(M)$,

$$
\begin{gathered}
\cdots \stackrel{F_{r+2}}{\longrightarrow} A^{s_{r+1}} \stackrel{F_{r+1}}{\longrightarrow} A^{s_{r}} \stackrel{F_{r}}{\longrightarrow} A^{s_{r-1}} \stackrel{F_{r-1}}{\longrightarrow} \cdots \stackrel{F_{2}}{\longrightarrow} A^{s_{1}} \stackrel{F_{1}}{\longrightarrow} A^{s_{0}} \stackrel{F_{0}}{\longrightarrow} \\
A^{s} / \operatorname{Syz}(M) \longrightarrow 0 .
\end{gathered}
$$

Thus, using syzygies we can compute the matrices $F_{r}$, for $r \geq 1$.

Step 3. We consider the complex

$$
\begin{gathered}
0 \longrightarrow \operatorname{Hom}_{A}\left(A^{s_{0}}, A^{t} / \operatorname{Syz}(N)\right) \stackrel{F_{1}^{*}}{\longrightarrow} \cdots \stackrel{F_{r}^{*}}{\longrightarrow} \operatorname{Hom}_{A}\left(A^{s_{r}}, A^{t} / \operatorname{Syz}(N)\right) \stackrel{F_{r+1}^{*}}{\longrightarrow} \\
\operatorname{Hom}_{A}\left(A^{s_{r+1}}, A^{t} / \operatorname{Syz}(N)\right) \stackrel{F_{r+2}^{*}}{\longrightarrow} \cdots
\end{gathered}
$$

and we recall that

$$
\operatorname{Ext}_{A}^{r}(M, N) \cong \operatorname{Ext}_{A}^{r}\left(A^{s} / \operatorname{Syz}(M), A^{t} / \operatorname{Syz}(N)\right)=\operatorname{ker}\left(F_{r+1}^{*}\right) / \operatorname{Im}\left(F_{r}^{*}\right) .
$$

However, by (3.6), for each $r \geq 1$ a presentation of $\operatorname{Hom}_{A}\left(A^{s_{r}}, A^{t} / \operatorname{Syz}(N)\right)$ is

$$
\operatorname{Hom}_{A}\left(A^{s_{r}}, A^{t} / \operatorname{Syz}(N)\right) \cong A^{t s_{r}} /<I_{s_{r}} \otimes S y z(N)>.
$$


Step 4. We compute the matrices $F^{*}$ in the following way: as we saw in (2.2), we have the following commutative diagram

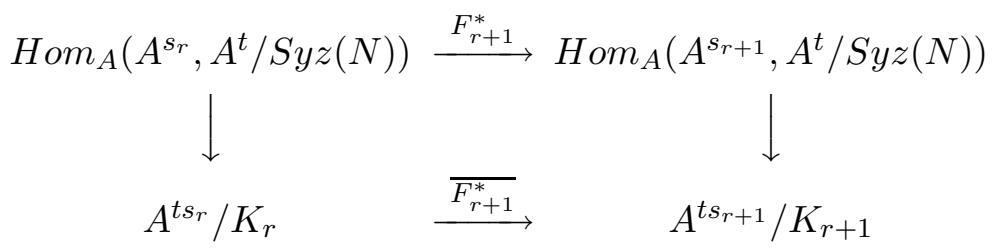

where $K_{r}=<I_{s_{r}} \otimes S y z(N)>$ and vertical arrows are isomorphisms obtained concatenating the columns of matrices of $\operatorname{Hom}_{A}\left(A^{s_{r}}, A^{t} / \operatorname{Syz}(N)\right)$, moreover, the matrices of homomorphisms $F_{r+1}^{*}$ and $\overline{F_{r+1}^{*}}$ coincides. So, we can replace the above complex for the following equivalent complex

$$
0 \longrightarrow A^{t s_{0}} / K_{0} \stackrel{F_{1}^{*}}{\longrightarrow} \cdots \stackrel{F_{r}^{*}}{\longrightarrow} A^{t s_{r}} / K_{r} \stackrel{F_{r+1}^{*}}{\longrightarrow} A^{t s_{r+1}} / K_{r+1} \stackrel{F_{r+2}^{*}}{\longrightarrow} \cdots .
$$

We will compute the matrix $F_{r+1}^{*}$ : let $\left\{\boldsymbol{e}_{1}, \ldots, \boldsymbol{e}_{t s_{r}}\right\}$ be the canonical basis of $A^{t s_{r}}$, then for each $1 \leq i \leq t s_{r}$, the element $\overline{\boldsymbol{e}_{i}}=\boldsymbol{e}_{i}+K_{r}$ can be replaced by its corresponding canonical matrix $G_{i}$, and since $F_{r+1}^{*}(G)=G F_{r+1}$ for each $G \in \operatorname{Hom}_{A}\left(A^{s_{r}}, A^{t} / S y z(N)\right)$, then we conclude that

$$
F_{r+1}^{*}=I_{t} \otimes F_{r+1}^{T} \text {. }
$$

Step 5. By (4.1), a presentation of $\operatorname{Ext}_{A}^{r}(M, N)$ is given by a presentation of $\operatorname{ker}\left(F_{r+1}^{*}\right) / \operatorname{Im}\left(F_{r}^{*}\right)$. Hence, we can apply the Theorem 7 , let $p_{r}$ be the number of generators of $\operatorname{ker}\left(F_{r+1}^{*}\right)=\operatorname{Syz}\left(F_{r+1}^{*}\right)=S y z\left(I_{t} \otimes F_{r+1}^{T}\right)$, we know how to compute this syzygy, and hence, we know how to compute $p_{r}$. We also know how to compute the matrix $F_{r}^{*}=I_{t} \otimes F_{r}^{T}$. Then, a presentation of $\operatorname{Ext}_{A}^{r}(M, N)$ is given by

$$
\operatorname{Ext}_{A}^{r}(M, N) \cong A^{p_{r}} / \operatorname{Syz}\left(\operatorname{ker}\left(F_{r+1}^{*}\right) / \operatorname{Im}\left(F_{r}^{*}\right)\right),
$$

where a set of generators of $\operatorname{Syz}\left(\operatorname{ker}\left(F_{r+1}^{*}\right) / \operatorname{Im}\left(F_{r}^{*}\right)\right)$ are the first $p_{r}$ coordinates of the generators of

$$
\left.\operatorname{Syz}\left[\operatorname{ker}\left(F_{r+1}^{*}\right) \mid \operatorname{Im}\left(F_{r}^{*}\right)\right)\right]=\operatorname{Syz}\left[\operatorname{Syz}\left[I_{t} \otimes F_{r+1}^{T}\right] \mid I_{t} \otimes F_{r}^{T}\right] .
$$

System of generators of $\operatorname{Ext}_{A}^{r}(M, N), r \geq 1$ : By (4.1), a system of generators for $\operatorname{Ext}_{A}^{r}(M, N)$ is defined by a system of generators of $\operatorname{ker}\left(F_{r+1}^{*}\right)=$ $\operatorname{Syz}\left(F_{r+1}^{*}\right)=\operatorname{Syz}\left(I_{t} \otimes F_{r+1}^{T}\right)$, hence if

$$
S y z\left[I_{t} \otimes F_{r+1}^{T}\right]=\left[\boldsymbol{h}_{1} \cdots \boldsymbol{h}_{p_{r}}\right]
$$

then

$$
\operatorname{Ext}_{A}^{r}(M, N)=<\widetilde{\boldsymbol{h}_{1}}, \ldots, \widetilde{\boldsymbol{h}_{p_{r}}}>
$$


where $\widetilde{\boldsymbol{h}_{u}}=\boldsymbol{h}_{u}+\operatorname{Im}\left(F_{r}^{*}\right), 1 \leq u \leq p_{r}$.

In the next example we will illustrate these procedures.

Example 21. Let $M$ and $N$ be submodules as in the Example 6, we will compute $\operatorname{Ext}_{M}^{r}(M, N)$, for $r \geq 1$. We will use the free resolution of $M$ that we computed in the Example 20. For $r=1$, we compute

$$
S y z\left[I_{3} \otimes F_{2}^{T}\right]=S y z\left[\begin{array}{lll}
2 & 0 & 0 \\
0 & 2 & 0 \\
0 & 0 & 2
\end{array}\right]=\left[\begin{array}{lll}
5 & 0 & 0 \\
0 & 5 & 0 \\
0 & 0 & 5
\end{array}\right],
$$

and hence, the value of $p_{1}$ in (4.2) is 3 . Moreover,

$$
I_{3} \otimes F_{1}^{T}=\left[\begin{array}{cccccc}
5 y & 5 x & 0 & 0 & 0 & 0 \\
0 & 0 & 5 y & 5 x & 0 & 0 \\
0 & 0 & 0 & 0 & 5 y & 5 x
\end{array}\right] .
$$

Next we must compute

$$
\begin{aligned}
S_{1} & =S y z\left[S y z\left[I_{3} \otimes F_{2}^{T}\right] \mid I_{3} \otimes F_{1}^{T}\right]= \\
& =S y z\left[\begin{array}{ccccccccc}
5 & 0 & 0 & 5 y & 5 x & 0 & 0 & 0 & 0 \\
0 & 5 & 0 & 0 & 0 & 5 y & 5 x & 0 & 0 \\
0 & 0 & 5 & 0 & 0 & 0 & 0 & 5 y & 5 x
\end{array}\right],
\end{aligned}
$$

and we get that

$$
\left[\begin{array}{ccccccccccccccccccc}
2 & 9 y & 9 x & 0 & 0 & 0 & 0 & 0 & 0 & 0 & 0 & 0 & 0 & 0 & 0 & 0 & 0 & 0 \\
0 & 0 & 0 & 2 & 9 y & 9 x & 0 & 0 & 0 & 0 & 0 & 0 & 0 & 0 & 0 & 0 & 0 & 0 \\
0 & 0 & 0 & 0 & 0 & 0 & 2 & 9 y & 9 x & 0 & 0 & 0 & 0 & 0 & 0 & 0 & 0 & 0 \\
0 & 1 & 0 & 0 & 0 & 0 & 0 & 0 & 0 & 2 & 0 & x & 0 & 0 & 0 & 0 & 0 & 0 \\
0 & 0 & 1 & 0 & 0 & 0 & 0 & 0 & 0 & 0 & 2 & 9 y & 0 & 0 & 0 & 0 & 0 & 0 \\
0 & 0 & 0 & 0 & 1 & 0 & 0 & 0 & 0 & 0 & 0 & 0 & 2 & 0 & x & 0 & 0 & 0 \\
0 & 0 & 0 & 0 & 0 & 1 & 0 & 0 & 0 & 0 & 0 & 0 & 0 & 2 & 9 y & 0 & 0 & 0 \\
0 & 0 & 0 & 0 & 0 & 0 & 0 & 1 & 0 & 0 & 0 & 0 & 0 & 0 & 0 & 2 & 0 & x \\
0 & 0 & 0 & 0 & 0 & 0 & 0 & 0 & 1 & 0 & 0 & 0 & 0 & 0 & 0 & 0 & 2 & 9 y
\end{array}\right] .
$$

In this matrix we choose the first three entries of each column and we get the generators of $S_{1}$, moreover, since 9 is invertible in $\mathbb{Z}_{10}$, then

$$
\begin{aligned}
& \boldsymbol{u}_{1}=(2,0,0), \boldsymbol{u}_{2}=(y, 0,0), \boldsymbol{u}_{3}=(x, 0,0) \\
& \boldsymbol{u}_{4}=(0,2,0), \boldsymbol{u}_{5}=(0, y, 0), \boldsymbol{u}_{6}=(0, x, 0) \\
& \boldsymbol{u}_{7}=(0,0,2), \boldsymbol{u}_{8}=(0,0, y), \boldsymbol{u}_{9}=(0,0, x)
\end{aligned}
$$

Thus,

$$
\operatorname{Ext}_{A}^{1}(M, N) \cong A^{3} /<\boldsymbol{u}_{1}, \boldsymbol{u}_{2}, \boldsymbol{u}_{3}, \boldsymbol{u}_{4}, \boldsymbol{u}_{5}, \boldsymbol{u}_{6}, \boldsymbol{u}_{7}, \boldsymbol{u}_{8}, \boldsymbol{u}_{9}>
$$


Moreover,

$$
\operatorname{Ext}_{A}^{1}(M, N)=<\widetilde{\boldsymbol{h}_{1}}, \widetilde{\boldsymbol{h}_{2}}, \widetilde{\boldsymbol{h}_{3}}>,
$$

where

$$
\begin{gathered}
\widetilde{\boldsymbol{h}_{1}}=5 \boldsymbol{e}_{1}+<\left[I_{3} \otimes F_{1}^{T}\right]>\widetilde{\boldsymbol{h}_{2}}=5 \boldsymbol{e}_{2}+<\left[I_{3} \otimes F_{1}^{T}\right]>, \\
\widetilde{\boldsymbol{h}_{3}}=5 \boldsymbol{e}_{3}+<\left[I_{3} \otimes F_{1}^{T}\right]>.
\end{gathered}
$$

For $r=2$ we have

$$
S y z\left[I_{3} \otimes F_{3}^{T}\right]=S y z\left[\begin{array}{ccc}
5 & 0 & 0 \\
0 & 5 & 0 \\
0 & 0 & 5
\end{array}\right]=\left[\begin{array}{lll}
2 & 0 & 0 \\
0 & 2 & 0 \\
0 & 0 & 2
\end{array}\right],
$$

so $p_{2}=3$, moreover,

$$
I_{3} \otimes F_{2}^{T}=\left[\begin{array}{lll}
2 & 0 & 0 \\
0 & 2 & 0 \\
0 & 0 & 2
\end{array}\right]
$$

and hence $\operatorname{ker}\left(F_{3}^{*}\right)=\operatorname{Im}\left(F_{2}^{*}\right)$, i.e.,

$$
\operatorname{Ext}_{A}^{2}(M, N)=0 \text {. }
$$

We can check this result using (4.3), thus we compute

$$
S_{2}=S y z\left[S y z\left[I_{3} \otimes F_{3}^{T}\right] \mid I_{3} \otimes F_{2}^{T}\right]=S y z\left[\begin{array}{cccccc}
2 & 0 & 0 & 2 & 0 & 0 \\
0 & 2 & 0 & 0 & 2 & 0 \\
0 & 0 & 2 & 0 & 0 & 2
\end{array}\right],
$$

and we get that

$$
S_{2}=\left[\begin{array}{lllllllll}
5 & 9 & 0 & 0 & 0 & 0 & 0 & 0 & 0 \\
0 & 0 & 5 & 9 & 0 & 0 & 0 & 0 & 0 \\
0 & 0 & 0 & 0 & 5 & 9 & 0 & 0 & 0 \\
0 & 1 & 0 & 0 & 0 & 0 & 5 & 0 & 0 \\
0 & 0 & 0 & 1 & 0 & 0 & 0 & 5 & 0 \\
0 & 0 & 0 & 0 & 0 & 1 & 0 & 0 & 5
\end{array}\right]
$$

thus the generators of $S_{2}$ are

$$
\begin{aligned}
& \boldsymbol{v}_{1}=(5,0,0), \boldsymbol{v}_{2}=(9,0,0), \\
& \boldsymbol{v}_{3}=(0,5,0), \boldsymbol{v}_{4}=(0,9,0), \\
& \boldsymbol{v}_{5}=(0,0,5), \boldsymbol{v}_{6}=(0,0,9) .
\end{aligned}
$$

But, $\left\langle\boldsymbol{v}_{2}, \boldsymbol{v}_{4}, \boldsymbol{v}_{6}>=A^{3}\right.$, and hence, $\operatorname{Ext}_{A}^{2}(M, N) \cong A^{3} /<\boldsymbol{v}_{1}, \ldots, \boldsymbol{v}_{6}>=$ 0 . Moreover, $\operatorname{Ext}_{A}^{2}(M, N)=<\widetilde{\boldsymbol{l}_{1}}, \widetilde{\boldsymbol{l}_{2}}, \widetilde{\boldsymbol{l}_{3}}>$, where $\widetilde{\boldsymbol{l}_{1}}=2 \boldsymbol{e}_{1}+<\left[I_{3} \otimes F_{2}^{T}\right]>=$ $\widetilde{0}, \widetilde{\boldsymbol{l}_{2}}=2 \boldsymbol{e}_{2}+<\left[I_{3} \otimes F_{2}^{T}\right]>=\widetilde{0}$ and $\widetilde{\boldsymbol{l}_{3}}=2 \boldsymbol{e}_{3}+<\left[I_{3} \otimes F_{2}^{T}\right]>=\widetilde{0}$. 
For $r=3$ we have

$$
S y z\left[I_{3} \otimes F_{4}^{T}\right]=S y z\left[\begin{array}{lll}
2 & 0 & 0 \\
0 & 2 & 0 \\
0 & 0 & 2
\end{array}\right]=\left[\begin{array}{lll}
5 & 0 & 0 \\
0 & 5 & 0 \\
0 & 0 & 5
\end{array}\right],
$$

so $p_{2}=3$, moreover,

$$
I_{3} \otimes F_{3}^{T}=\left[\begin{array}{ccc}
5 & 0 & 0 \\
0 & 5 & 0 \\
0 & 0 & 5
\end{array}\right]
$$

and hence $\operatorname{ker}\left(F_{4}^{*}\right)=\operatorname{Im}\left(F_{3}^{*}\right)$, i.e.,

$$
\operatorname{Ext}_{A}^{3}(M, N)=0 .
$$

We can check this result using (4.3), thus we compute

$$
S_{3}=S y z\left[S y z\left[I_{3} \otimes F_{4}^{T}\right] \mid I_{3} \otimes F_{3}^{T}\right]=S y z\left[\begin{array}{cccccc}
5 & 0 & 0 & 5 & 0 & 0 \\
0 & 5 & 0 & 0 & 5 & 0 \\
0 & 0 & 5 & 0 & 0 & 5
\end{array}\right],
$$

and we get that

$$
S_{3}=\left[\begin{array}{lllllllll}
2 & 9 & 0 & 0 & 0 & 0 & 0 & 0 & 0 \\
0 & 0 & 2 & 9 & 0 & 0 & 0 & 0 & 0 \\
0 & 0 & 0 & 0 & 2 & 9 & 0 & 0 & 0 \\
0 & 1 & 0 & 0 & 0 & 0 & 2 & 0 & 0 \\
0 & 0 & 0 & 1 & 0 & 0 & 0 & 2 & 0 \\
0 & 0 & 0 & 0 & 0 & 1 & 0 & 0 & 2
\end{array}\right]
$$

thus the generators of $S_{3}$ are

$$
\begin{aligned}
& \boldsymbol{w}_{1}=(2,0,0), \boldsymbol{w}_{2}=(9,0,0), \\
& \boldsymbol{w}_{3}=(0,2,0), \boldsymbol{w}_{4}=(0,9,0), \\
& \boldsymbol{w}_{5}=(0,0,2), \boldsymbol{w}_{6}=(0,0,9) .
\end{aligned}
$$

But, $<\boldsymbol{w}_{2}, \boldsymbol{w}_{4}, \boldsymbol{w}_{6}>=A^{3}$, and hence, $\operatorname{Ext}_{A}^{3}(M, N) \cong A^{3} /<\boldsymbol{w}_{1}, \ldots, \boldsymbol{w}_{6}>$ $=0$. Moreover, $\operatorname{Ext}_{A}^{3}(M, N)=<\widetilde{\boldsymbol{m}_{1}}, \widetilde{\boldsymbol{m}_{2}}, \widetilde{\boldsymbol{m}_{3}}>$, where $\widetilde{\boldsymbol{m}_{1}}=5 \boldsymbol{e}_{1}+<$ $\left[I_{3} \otimes F_{3}^{T}\right]>=\widetilde{0}, \widetilde{\boldsymbol{m}_{2}}=5 \boldsymbol{e}_{2}+<\left[I_{3} \otimes F_{3}^{T}\right]>=\widetilde{0}$ and $\widetilde{\boldsymbol{m}_{3}}=5 \boldsymbol{e}_{3}+<$ $\left[I_{3} \otimes F_{3}^{T}\right]>=\widetilde{0}$.

We conclude that

$$
\operatorname{Ext}_{A}^{r}(M, N)=0, \text { for } r \geq 2 .
$$

4.2. Computation of Tor. Using syzygies and some previous results we now describe an easy procedure for computing the $A$-modules $\operatorname{Tor}_{r}^{A}(M, N)$ for $r \geq 0$, where $M=<\boldsymbol{f}_{1}, \ldots, \boldsymbol{f}_{s}>\subseteq A^{m}$ and $N=<\boldsymbol{g}_{1}, \ldots, \boldsymbol{g}_{t}>\subseteq A^{l}$. 
By computing we mean to find a presentation and a system of generators of $\operatorname{Tor}_{r}^{A}(M, N), r \geq 0$ (compare with [8], Proposition 7.1.3). For $r=0$, the computation is given by the Theorem 17 . So we assume that $r \geq 1$.

Presentation of $\operatorname{Tor}_{r}^{A}(M, N), r \geq 1$ :

Step 1. We compute presentations of $M$ and $N$,

$$
M \cong A^{s} / \operatorname{Syz}(M), N \cong A^{t} / \operatorname{Syz}(N),
$$

Step 2. We compute a free resolution of $A^{t} / \operatorname{Syz}(N)$ using theorem 19,

$$
\begin{gathered}
\cdots \stackrel{G_{r+2}}{\longrightarrow} A^{t_{r+1}} \stackrel{G_{r+1}}{\longrightarrow} A^{t_{r}} \stackrel{G_{r}}{\longrightarrow} A^{t_{r-1}} \stackrel{G_{r-1}}{\longrightarrow} \cdots \stackrel{G_{2}}{\longrightarrow} A^{t_{1}} \stackrel{G_{1}}{\longrightarrow} A^{t_{0}} \stackrel{G_{0}}{\longrightarrow} \\
A^{t} / \operatorname{Syz}(N) \longrightarrow 0 .
\end{gathered}
$$

Step 3. We consider the complex

$$
\begin{gathered}
\cdots \stackrel{i \otimes G_{r+2}}{\longrightarrow} A^{s} / \operatorname{Syz}(M) \otimes A^{t_{r+1}} \stackrel{i \otimes G_{r+1}}{\longrightarrow} A^{s} / \operatorname{Syz}(M) \otimes A^{t_{r}} \stackrel{i \otimes G_{r}}{\longrightarrow} \cdots \stackrel{i \otimes G_{2}}{\longrightarrow} \\
A^{s} / \operatorname{Syz}(M) \otimes A^{t_{1}} \stackrel{i \otimes G_{1}}{\longrightarrow} A^{s} / \operatorname{Syz}(M) \otimes A^{t_{0}} \longrightarrow 0,
\end{gathered}
$$

where $i$ is the identical of $A^{s} / \operatorname{Syz}(M)$ and then

$$
\begin{gathered}
\operatorname{Tor}_{r}^{A}(M, N) \cong \operatorname{Tor}_{r}^{A}\left(A^{s} / \operatorname{Syz}(M), A^{t} / \operatorname{Syz}(N)\right)= \\
\operatorname{ker}\left(1 \otimes G_{r}\right) / \operatorname{Im}\left(1 \otimes G_{r+1}\right),
\end{gathered}
$$

but the matrix of $i \otimes G_{r}$ is $I_{s} \otimes G_{r}$, so $\operatorname{ker}\left(i \otimes G_{r}\right)=\operatorname{ker}\left(I_{s} \otimes G_{r}\right)=$ $\operatorname{Syz}\left(I_{s} \otimes G_{r}\right)$ and we get

$$
\operatorname{Tor}_{r}^{A}(M, N) \cong \operatorname{ker}\left(I_{s} \otimes G_{r}\right) / \operatorname{Im}\left(I_{s} \otimes G_{r+1}\right) .
$$

Step 3. Let $q_{r}$ be the number of generators of $S y z\left(I_{s} \otimes G_{r}\right)$, then by the Theorem 7, a presentation of $\operatorname{Tor}_{r}^{A}(M, N)$ is given by

$$
\operatorname{Tor}_{r}^{A}(M, N) \cong A^{q_{r}} / \operatorname{Syz}\left(\operatorname{ker}\left(I_{s} \otimes G_{r}\right) / \operatorname{Im}\left(I_{s} \otimes G_{r+1}\right)\right),
$$

where a set of generators of $\operatorname{Syz}\left(\operatorname{ker}\left(I_{s} \otimes G_{r}\right) / \operatorname{Im}\left(I_{s} \otimes G_{r+1}\right)\right)$ are the first $q_{r}$ coordinates of generators of

$$
\operatorname{Syz}\left[\operatorname{Syz}\left[I_{s} \otimes G_{r}\right] \mid I_{s} \otimes G_{r+1}\right] .
$$

System of generators of $\operatorname{Tor}_{r}^{A}(M, N), r \geq 1$ : By (4.4), a system of generators of $\operatorname{Tor}_{r}^{A}(M, N)$ is given by a system of generators of $\operatorname{ker}\left(i \otimes G_{r}\right)=$ $\operatorname{Syz}\left(I_{s} \otimes G_{r}\right)$. Thus, if

$$
\operatorname{Syz}\left[I_{s} \otimes G_{r}\right]=\left[\boldsymbol{h}_{1} \cdots \boldsymbol{h}_{q_{r}}\right],
$$

then

$$
\operatorname{Tor}_{r}^{A}(M, N)=<\widetilde{\boldsymbol{h}_{1}}, \ldots, \widetilde{\boldsymbol{h}_{q_{r}}}>
$$


where $\widetilde{\boldsymbol{h}_{v}}=\boldsymbol{h}_{v}+\operatorname{Im}\left(I_{s} \otimes F G_{r+1}\right), 1 \leq v \leq q_{r}$.

In the next example we will illustrate these procedures.

Example 22. Let $M$ and $N$ be submodules as in the Example 6, we will compute $\operatorname{Tor}_{r}^{A}(M, N)$, for $r \geq 1$. We will use the free resolution of $N$ that we computed in the Example 20. For $r=1$, we compute

$$
\begin{aligned}
& \operatorname{ker}\left(I_{2} \otimes G_{1}\right)=S y z\left[I_{2} \otimes G_{1}\right]=S y z\left[\begin{array}{cccc}
5 & 2 x+9 y & 0 & 0 \\
0 & 8 x & 0 & 0 \\
5 & y & 0 & 0 \\
0 & 0 & 5 & 2 x+9 y \\
0 & 0 & 0 & 8 x \\
0 & 0 & 5 & y
\end{array}\right]= \\
& {\left[\begin{array}{llll}
2 & y & 0 & 0 \\
0 & 5 & 0 & 0 \\
0 & 0 & 2 & y \\
0 & 0 & 0 & 5
\end{array}\right],}
\end{aligned}
$$

but

$$
I_{2} \otimes G_{2}=\left[\begin{array}{cccc}
2 & y & 0 & 0 \\
0 & 5 & 0 & 0 \\
0 & 0 & 2 & y \\
0 & 0 & 0 & 5
\end{array}\right]
$$

hence $\operatorname{ker}\left(I_{2} \otimes G_{1}\right)=\operatorname{Im}\left(I_{2} \otimes G_{2}\right)$, and by (4.4),

$$
\operatorname{Tor}_{1}^{A}(M, N)=0 \text {. }
$$

For $r=2$ we have

$$
\operatorname{ker}\left(I_{2} \otimes G_{2}\right)=S y z\left[I_{2} \otimes G_{2}\right]=S y z\left[\begin{array}{cccc}
2 & y & 0 & 0 \\
0 & 5 & 0 & 0 \\
0 & 0 & 2 & y \\
0 & 0 & 0 & 5
\end{array}\right]=\left[\begin{array}{cccc}
5 & y & 0 & 0 \\
0 & 8 & 0 & 0 \\
0 & 0 & 5 & y \\
0 & 0 & 0 & 8
\end{array}\right],
$$

but

$$
I_{2} \otimes G_{3}=\left[\begin{array}{cccc}
5 & y & 0 & 0 \\
0 & 8 & 0 & 0 \\
0 & 0 & 5 & y \\
0 & 0 & 0 & 8
\end{array}\right]
$$

hence

$$
\operatorname{Tor}_{2}^{A}(M, N)=0 \text {. }
$$

We conclude that

$$
\operatorname{Tor}_{r}^{A}(M, N)=0, \text { for } r \geq 1 .
$$


It is well known (see [16]) that $\operatorname{Tor}_{r}^{A}(M, N)$ could be also computed using a free resolution of $M$. In this case (4.6) should be replaced by

$$
S y z\left[S y z\left[F_{r} \otimes I_{t}\right] \mid F_{r+1} \otimes I_{t}\right] .
$$

So, for $r=1$ we have

$$
\operatorname{ker}\left(F_{1} \otimes I_{3}\right)=S y z\left[F_{1} \otimes I_{3}\right]=S y z\left[\begin{array}{ccc}
5 y & 0 & 0 \\
0 & 5 y & 0 \\
0 & 0 & 5 y \\
5 x & 0 & 0 \\
0 & 5 x & 0 \\
0 & 0 & 5 x
\end{array}\right]=\left[\begin{array}{ccc}
2 & 0 & 0 \\
0 & 2 & 0 \\
0 & 0 & 2
\end{array}\right],
$$

but

$$
F_{2} \otimes I_{3}=\left[\begin{array}{lll}
2 & 0 & 0 \\
0 & 2 & 0 \\
0 & 0 & 2
\end{array}\right]
$$

hence $\operatorname{ker}\left(F_{1} \otimes I_{3}\right)=\operatorname{Im}\left(F_{2} \otimes I_{3}\right)$ and

$$
\operatorname{Tor}_{1}^{A}(M, N)=0 \text {. }
$$

For $r=2$ we have

$$
\operatorname{ker}\left(F_{2} \otimes I_{3}\right)=S y z\left[F_{2} \otimes I_{3}\right]=S y z\left[\begin{array}{lll}
2 & 0 & 0 \\
0 & 2 & 0 \\
0 & 0 & 2
\end{array}\right]=\left[\begin{array}{ccc}
5 & 0 & 0 \\
0 & 5 & 0 \\
0 & 0 & 5
\end{array}\right],
$$

but

$$
F_{3} \otimes I_{3}=\left[\begin{array}{lll}
5 & 0 & 0 \\
0 & 5 & 0 \\
0 & 0 & 5
\end{array}\right]
$$

hence

$$
\operatorname{Tor}_{2}^{A}(M, N)=0
$$

We conclude again that

$$
\operatorname{Tor}_{r}^{A}(M, N)=0, \text { for } r \geq 1 .
$$

\section{References}

[1] Adams, W.W. and Loustaunau, P., An Introduction to Gröbner Bases, Graduate Studies in Mathematics, AMS, 1994.

[2] Becker, T. and Weispfenning, V., Gröbner Bases, Graduate Texts in Mathematics, vol. 141, Springer, 1993. 
[3] Buchberger, B., Ein Algorithmus zum Auffinden der Basiselemente des Restklassenringes nach einem nulldimensionalen Polynomideal, Ph.D. Thesis, Inst. University of Innsbruck, Innsbruck, Austria, 1965.

[4] Buchberger, B. and Winkler, F., Gröbner Bases and Applications, London Mathematical Society, Lecture Notes Series, 251, Cambridge University Press, 1998.

[5] Chyzak, F., Quadrat, A. and Robertz, D., Effective algorithms for parametrizing linear control systems over Ore algebras, INRIA, Rapport de recherche $\mathrm{n}^{\circ} 5181,2004$.

[6] Cox, D., Little, J. and O'Shea, D., Ideals, Varieties and Algoritms, Springer, Corr. 5th printing, 2006.

[7] Cox, D., Little, J. and O'Shea, D., Using Algebraic Geometry, Springer, 1998.

[8] Greuel, G. and Pfister, G., A Singular Introduction to Commutative Algebra, Springer, 2002

[9] http://cocoa.dima.unige.it

[10] http://www.singular.uni-kl.de

[11] Kreuzer, M. and Robbiano, L., Computational Commutative Algebra 1, Springer, 2000.

[12] Kreuzer, M. and Robbiano, L., Computational Commutative Algebra 2, Springer, 2005.

[13] Lezama, O., Gröbner bases for modules over Noetherian polynomial commutative rings, Georgian Mathematical Journal, Vol. 15, 2008, 121-137.

[14] Quadrat, A. and Pommaret, J.F., Localization and parametrization of linear multidimensional control systems, Systems \& Control Letters, 37, 1999, 247-260.

[15] Rutman, E.W., Gröbner bases and primary decomposition of modules, J. Symb. Comp., 14, 1992, 483-503.

[16] Vermani, L.R., An Elementary Approach to Homological Algebra, Chapman \& Hall/CRC, Monographs and Surveys in Pure and Applied Mathematics 130, 2003. 